\title{
Selection and evaluation of reference genes for analysis of mouse (Mus musculus) sex-dimorphic brain development
}

\author{
Tanya T Cheung $^{1}{ }^{1}$, Mitchell K Weston ${ }^{1}$, Megan J Wilson ${ }^{\text {Corresp. }} 1$ \\ 1 Department of Anatomy, University of Otago, Dunedin, New Zealand \\ Corresponding Author: Megan J Wilson \\ Email address: meganj.wilson@otago.ac.nz
}

The development of the brain is sex-dimorphic, and as a result so are many neurological disorders. One approach for studying sex-dimorphic brain development is to measure gene expression in biological samples using RT-qPCR. However, the accuracy and consistency of this technique relies on the reference gene(s) selected. We analyzed the expression of ten reference genes in male and female samples over three stages of brain development, using popular algorithms NormFinder, GeNorm and Bestkeeper. The top ranked reference genes at each time point were further used to quantify gene expression of three sexdimorphic genes (Wnt10b, Xist and CYP7B1). When comparing gene expression between the sexes expression at specific time points the best reference gene combinations are: Sdha/Pgk1 at E11.5, RpL38/Sdha E12.5, and Actb/RpL37 at E15.5. When studying expression across time, the ideal reference gene(s) differs with sex. For XY samples a combination of $A c t b / S d h a$. In contrast, when studying gene expression across developmental stage with XX samples, Sdha/Gapdh were the top reference genes. Our results identify the best combination of two reference genes when studying male and female brain development, and emphasize the importance of selecting the correct reference genes for comparisons between developmental stages. 
1

2

3

4

17 Dunedin 9054, New Zealand

$18 *$ Corresponding author

19 Email: meganj.wilson@otago.ac.nz.Ph: +64 3 4704695, Fax: +64 4797254 


\section{Abstract}

22 The development of the brain is sex-dimorphic, and as a result so are many neurological

23 disorders. One approach for studying sex-dimorphic brain development is to measure gene

24 expression in biological samples using RT-qPCR.

25 However, the accuracy and consistency of this technique relies on the reference gene(s)

26 selected.

27 We analyzed the expression of ten reference genes in male and female samples over three 28 stages of brain development, using popular algorithms NormFinder,

29 GeNorm and Bestkeeper. The top ranked reference genes at each time point were further used to quantify gene expression of three sex-dimorphic genes (Wnt10b,

31 Xist and CYP7B1).

32 When comparing gene expression between the sexes expression at specific time

33 points the best reference gene combinations are: Sdha/Pgk1 at E11.5, RpL38/Sdha E12.5, 34 and $A c t b / R p L 37$ at E15.5. When studying expression across time, the ideal reference 35 gene(s) differs with sex. For XY samples a combination of Actb/Sdha. In contrast, when 36 studying gene expression across developmental stage with XX samples, Sdha/Gapdh were 37 the top reference genes.

38 Our results identify the best combination of two reference genes when studying male and female brain development, and emphasize the importance of selecting the correct reference genes for comparisons between developmental stages. 


\section{Introduction}

50 Mammals that reproduce through sexual reproduction have intrinsic differences between

51 male and female sexes. The most obvious morphological differences are those of 52 reproductive organs. However, many other less apparent organs and tissues are also 53 modulated in a sex-dimorphic manner. In particular, there exist numerous differences in 54 the human brain between the male and female sexes. The rate of maturation of the female 55 brain reaches its peak maturity at 10 years of age compared to the male brain, which only 56 peaks 4 years later (Giedd et al., 1997). On average females have a higher percentage of 57 gray matter although volume is smaller in comparison (Goldstein et al., 2001; $\underline{\text { Gur et al., }}$ 58 1999) neuronal cells in the hypothalamus may only be responsive to estrogen or 59 testosterone (Arnold, 2004; Lenroot et al., 2007). Furthermore, numerous neurological 60 diseases have sex-specific biases (Fombonne, 2009), including, schizophrenia (risk ratio 1 61 female:1.4 male) (Abel et al., 2010; Saha et al., 2005) and Autism spectrum disorder (1 62 female:4 male) (Mottron et al., 2015). In order to understand how such differences arise at 63 the level of gene expression, we set out to determine the best set of reference genes to 64 study mouse sex-dimorphic brain development during key developmental stages.

66 The house mouse (Mus musculus) is an excellent choice for embryonic studies of 67 mammalian development owing to its comparatively short gestation period and 68 accelerated life span. In particular, mice have been widely used to study sex-dimorphic 69 brain development (Maekawa et al., 2014; Ngun et al., 2011). However to acquire more 70 insight into the molecular drives of sex-dimorphic brain development, it is necessary to 
71 study the expression of genes in the developing brain. High-throughput sequencing

72 technologies such as RNA-sequencing provide a powerful technique to study changes in

73 gene expression (Hrdlickova et al., 2016). However, data gathered from these high-

74 throughput technologies needs to be validated to ensure accurate interpretation through

75 repeated biological replicates.

76

77 The most commonly used method for validating the expression of a gene identified by 78 sequencing is Reverse Transcription quantitative Polymerase Chain Reaction (RT-qPCR).

79 RT-qPCR allows for the detection and quantification of specific cDNA fragments generated 80 from RNA samples. However, to obtain levels of expression comparable between samples,

81 the target gene must be normalized to the expression of at least two internal controls 82 (termed reference genes) that are stably expressed throughout all samples. Normalization

83 is needed to compensate for different amounts of cDNA present in the sample along with 84 differing PCR efficiencies of primer sets. Therefore, the selection of the reference gene is 85 important as inappropriate reference genes can bias the data and thus lead to 86 misinterpretation of results.

87

Ideally, the reference gene should be present at a consistent level across all compared samples, regardless of treatment or disease state of the sample. Furthermore, the chosen reference gene should be constitutively expressed across all cell types and tissues.

91 However despite large-scale high-throughput technologies, no such gene has been found.

92 Therefore, the most common and validated approach is to find a reference gene that is the

93 least variable in the specific context of the study. 
95 The most regularly used reference genes are Actb and Gapdh (Boda et al., 2009). However, 96 a number of studies have shown that these genes are expressed differentially in the brain. 97 Gapdh has been shown to have sex dimorphic protein levels in adulthood (Perrot-Sinal et 98 al., 2001) and to be up regulated in neuronal apoptosis (Chen et al., 1999; $\underline{\text { Sawa et al., }}$ 99 1997). Actb and Gapdh expression has also been shown to vary across tissue types, among 100 cell types and also during stages of cell proliferation and development of the brain (Sotelo101 Silveira et al., 2008; Veazey and Golding, 2011).

102

103 To fill in the gap in the literature, we set out to find a set of reference genes most suitable 104 for studies on the embryonic brain from E11.5 to E15.5 in males and females. This work 105 will prove highly valuable unbiased study to uncover suitable reference genes for studies of 106 embryonic brain development and illustrate the importance of the accurate selection of 107 reference genes for RT-qPCR analyses.

Methods

110 All animal work was performed under the University of Otago Animal Ethics Committee 111 number: ET13/14. Inbred C57BL/6 mice were purchased from the Hercus Taieri Resource 112 Unit (University of Otago, Dunedin, NZ).

114 Sample collection

115 Whole embryonic brain tissue (overlying epidermis and cranial facial tissues were 116 removed) and tail tips were collected from timed stages (E11.5 to 18.5) pregnant mothers. 
117 RNA was extracted from brain tissue using Purelink RNA mini kit (Ambion, USA) according

118 to manufacturer's instructions. DNA was isolated from tail tips using $0.2 \mathrm{mg} / \mathrm{mL}$ (final

119 concentration) Proteinase K (New England Biolabs, Massachusetts, USA) and then added to

120 DirectPCR Lysis reagent 102-T (Viagen Biotechnologies, CA). Tail tips were incubated

121 overnight at $55^{\circ} \mathrm{C}$ and followed by heat inactivation of the proteinase $\mathrm{K}$ at $85^{\circ} \mathrm{C}$ for $45 \mathrm{~min}$.

122 Samples were centrifuged at $14,000 \mathrm{~g}$ for $1 \mathrm{~min}$ to pellet cell debris and $2 \mu \mathrm{L}$ of each sample

123 was used for sexing PCR. The RNA for each time point and sex was a pooled sample, with a

124 minimum of three separate biological samples collected for each condition (from separate

125 litters). Total RNA was quantified with a Nanodrop (ThermoFisher, USA) and purity

126 assessed using the $260 / 230$ and $260 / 280$ ratios. A ratio of $\sim 2$ was taken as acceptable for

127 pure RNA.

128

129 Sexing of embryos by PCR

130 To sex the embryos, the Sry gene was amplified using primers listed in Supplementary

131 Table 1. Following PCR, products were run on a $2 \%$ agarose gel, a band appears at 132 approximately $380 \mathrm{bp}$, indicating the presence of the Y-chromosome for male embryos.

133

134 DNase treatment and reverse transcription

135 To remove genomic DNA present in the sample, $1 \mu \mathrm{g}$ RNA was added to $1 \mu \mathrm{L}$ DNase and 136 incubated at $37^{\circ} \mathrm{C}$ for $40 \mathrm{~min}$. A phenol/chloroform extraction was carried out followed by 137 ethanol precipitation to purify sample. Reverse transcriptase was performed using iScript 138 (Bio-Rad, California, USA) according to manufacturer's instructions. 
$140 \quad R T-q P C R$

141 Oligonucleotide primers (Supplementary Table 1) were designed and ordered from

142 Integrated DNA Technologies (IDT, USA). Each reaction contained $10 \mu \mathrm{L}$ SYBR Select

143 Master Mix (ThermoFisher, USA), $1.25 \mu \mathrm{L} 20$ pmol forward and reverse primers, $6.75 \mu \mathrm{L}$

144 water and $2 \mu \mathrm{L}$ cDNA. All reactions also included a no reverse transcription control and

145 each reaction was carried out in triplicate. RT-qPCR was carried out in the Stratagene

146 Mx3000p (Agilent technologies, California, USA) under the following conditions:

147 denaturing at $50{ }^{\circ} \mathrm{C}$ for $2 \mathrm{~min}$, annealing $96{ }^{\circ} \mathrm{C}$ for $2 \mathrm{~min}$, followed by 40 cycles of

148 amplification $\left(96^{\circ} \mathrm{C} 15 \mathrm{~s}, 60^{\circ} \mathrm{C} 15 \mathrm{~s}\right.$ and $\left.72^{\circ} \mathrm{C} 1 \mathrm{~min}\right)$. A final cycle for melt curve analysis

149 was included for every qPCR plate (one cycle: $95{ }^{\circ} \mathrm{C} 1 \mathrm{~min}, 55^{\circ} \mathrm{C} 30 \mathrm{~s}$ and $95{ }^{\circ} \mathrm{C} 30 \mathrm{~s}$ ). The

150 threshold was automatically set by the MXPro program (v. 4.10), and the Ct value for each

151 sample was calculated from the average of three technical replicates.

152

153 Data analysis

154 Data from RT-qPCR was then analyzed using three algorithms to calculate the most stable 155 or best suited reference gene; NormFinder (Andersen et al. 2004), GeNorm with SLqPCR R156 based package (Hellemans, 2007), Bestkeeper (Pfaffl, 2004) and RefFinder (deltaCt 157 method, (Silver et al., 2006; Xie et al., 2012) software. All data was analyzed according to 158 the program instructions.

159

160 The geometric mean was calculated for each reference gene by assigning a number (1-5)

161 for top ranked genes for each algorithm. The following equation was used to calculate the 
162 geometric mean for each data set: $\sqrt[3]{ }$ NormFinder*GeNorm*BestKeeper*deltaCt. The 163 reference genes were then ranked again based on their lowest geometric mean.

164

165 Analysis of sex specific gene expression was calculated using the following equation: $\Delta \mathrm{Ct}=2^{-}$

166 (Ctgene-Ctreference) with the average Ct value for the top two ranked reference genes. Statistical

167 testing used was either the unpaired Student's t-test when comparing two time-points or a 168 2-way ANOVA when comparing multiple time-points across time (Tukey's multiple 169 comparison test).

170

171 Results

172 Selection of candidate reference genes and mRNA transcript levels

173 To ensure accurate analysis of gene expression, ten candidate reference genes (Table 1)

174 were investigated to determine how stable each gene is for sex and time-point of 175 development. The candidate reference genes selected included those genes commonly used

176 in RT-qPCR experiments (such as Gapdh and Actb) and genes were stably expressed in 177 multiple mouse adult tissues, but had not been tested for suitability as reference genes with 178 mouse embryo tissues (Kouadjo et al., 2007). RT-qPCR was carried out for brain tissue 179 samples across three time points: E11.5, E12.5 and E15.5 using both male and female 180 samples. These time points were chosen for analysis as the sex determination in mouse 181 occurs between E11.0 and E12.0. During this $24 \mathrm{~h}$ period, considerable changes also occur 182 with respect to neuronal development, in particular, the formation of the primary brain

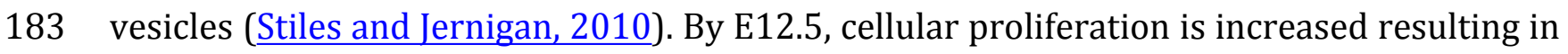
184 the expansion of neuronal precursors and formation of cortical layers (Finlay and 
185 Darlington, 1995). Neuronal differentiation, axonal branching and synaptogenesis is taking 186 place around day 15.5 (summarized in Fig. 1) (Sur and Rubenstein, 2005). The raw Ct 187 values for each reference gene across time and each sex are plotted in figure 2.

188

189

Determination of best-suited reference genes for male and female developing brain.

190 Four algorithms: NormFinder, GeNorm, BestKeeper and comparative deltaCt were 191 employed to determine the best-suited reference genes for either specific developmental 192 stage or between sex over time. Female and male samples were combined to identify the 193 most stable reference gene across time. At each developmental stage (E11.5, E12.5 and 194 E15.5) female and male samples were compared against each reference gene to determine 195 the best reference gene between both sexes.

196

197 The raw Ct values for each gene comparing sex-expression at each time point are shown as 198 box and whisker plots (Fig. 2) with minimum and maximum values indicated at each time 199 point. Ct values for male samples ranged between Ct values of 15-25.5 and female samples 200 between 15-28 (Supplementary Table 3). Across all time points, the reference gene with 201 least amount of variability for male samples is Actb with a mean Ct value of $16.36(+/-$ 202 1.178 SD) while the highest is Eif3 $f$ with a mean Ct value of 30.06 (+/- 5.08 SD). In females, 203 Actb mean Ct value is $17.9(+/-2.22 \mathrm{SD})$ and Eef2 has a mean Ct of 22.53 , while this Ct value 204 was not the highest, the SD had the highest amount of variability of +/- 4.54 SD. 
207 NormFinder Excel based add-on is an algorithm created by Andersen et al. (2004) that 208 ranks a set of candidate reference genes using stability values according to the variation in 209 expression across samples and between groups. A low stability value represents a 210 reference gene with the most stable expression in a given sample set. NormFinder analysis

211 identified, that across all developmental stages of development (Fig. 3A, Supplementary 212 Table 4), the reference gene with the lowest stability value was $R p L 37$ (1.211), the best 213 combination of genes were Hprt1 and RpL38 (0.559) in the male embryonic brain. In 214 female samples, Hprt1 (0.929) was the most stable gene across all time points, the best two 215 gene combination was Sdha and RpL38 (stability value of 0.640) (Fig. 3A). NormFinder 216 identified the lowest stability value at E11.5 to be Actb (0.309) (Fig. 3B), however Actb and 217 Sdha (0.250) genes gave the best combined stability value (0.250) for RT-qPCR analysis. 218 Following sex determination of the embryo, $R p L 38$ (0.253) was the most stable reference 219 gene at E12.5, with a combination of $R p L 38$ and $R p L 37$ (0.189) for use of two reference 220 genes (Fig. 3B). RpL37 (0.145) is the most stable reference gene at E15.5 however for 221 analyses with two reference genes then Pgk1 and Eef2 (0.430) were recommended 222 (Supplementary Table 3).

224 BestKeeper software determines the ideal reference genes out of a number of candidates

225 based on the standard error (+/- CP) of Ct values and combines them into an index using 226 repeated pair-wise correlation analysis (Pfaffl et al., 2004). Across all developmental 227 stages, the BestKeeper identified Pgk1 (SE: +/- 1.064) and Sdha (SE: +/- 1.004) as the best 
228 reference gene in the male sample, while Gapdh (SE: +/- 1.639) and Sdha (SE: +/- 1.441) to

229 be ideal for female samples (Fig. 3C). However at specific developmental stages, the ideal 230 reference gene differs. At E11.5 RpL38 (SE: +/- 0.68) is ranked the top reference gene, 231 while Pgk1 (SE: +/- 0.522) and Hprt1 (SE: +/- 1.762) are the most stable reference genes 232 for E12.5 and E15.5 respectively (Fig, 3D, Supplementary Table 5).

234 The SLqPCR R-based package uses the GeNorm algorithm (Hellemans et al., 2007) which 235 determines the most stable reference gene from a set of samples by calculating the 236 geometric mean of each reference gene in a stepwise calculation. A low M value indicates 237 stable expression of the gene: for homogenous samples a value below 0.5 indicates an 238 unstable reference gene in the samples analyzed, whereas for heterogeneous tissues the 239 mean M value below 1 is acceptable (Hellemans et al., 2007). The best stability value for the 240 female samples across all time points was Sdha and Gapdh with a stability M value of 0.591 241 while the top three most stable reference genes in the male samples were Pgk1, and ActB 242 with a mean M stability value of 0.281 (Fig. 4A, Supplementary Table 6).

244 At specific developmental time points, Sdha and Pgk1 (M of 0.2470) were calculated as the 245 most stable reference genes for analysis of male and female samples at E11.5. Following 246 sex determination in both male and female samples, Actb and Gapdh (M of 0.346) gene are 247 the most stable (Fig. 4B). During increased neuronal proliferation at E15.5, Actb and Pgk1 248 ( $\mathrm{M}$ of 0.719 ) gave the lowest $\mathrm{M}$ values, indicating these are reliable reference genes 
249 according to GeNorm analysis given at this stage the developing brain is heterogeneous 250 (Supplementary Table 6).

252 A forth method, termed the comparative deltaCt method (Silver et al., 2006) was also used 253 to compare reference genes. This uses a method similar to that of GeNorm, determining the 254 variation of gene expression between paired putative reference genes (the deltaCt) within 255 each sample. Variable deltaCt values for each pair between multiple samples, resulting in a 256 high standard deviation when the average deltaCt is calculated, means either gene or both 257 are not stably expressed. Pairs at E11.5 were Pgk1 and Sdha (1.98/1.87), Actb and Pgk1 258 (1.98/1.82). At E15.5 there was much more variation between reference genes, best pair 259 were Actb and Sdha (4.7) (Fig. 4D; Supplementary Table 7). When considering brain 260 development overtime, for the male samples Pgk1 and Sdha (2.1) were the best pair, 261 whereas for female samples, Hprt1 and Sdha (2.7) had the lowest stand deviation of deltaCt 262 values (Fig. 4C).

264 Geometric mean of each reference gene

265 Each reference gene was ranked from most stable to least stable, as determined by the four 266 stability calculations described above, and assigned a number from 1 (most stable) to 10 267 (least stable) (Supplementary Table 8). The geometric mean was taken for each set of 268 rankings to calculate an overall 'best' reference gene for each condition (Table 2 and 3). 269 The recommended reference genes across all stages in both male and female samples are 
270 Sdha and RpL37 (GeoMean: 1.18 and 2.94 respectively). Across all stages of embryonic

271 development in males, the recommended reference genes are Actb and Sdha (GeoMean:

2722.73 and 1.96) while in the female brain, the most stable set of reference genes are Sdha

273 and Gapdh (GeoMean 1.2 and 2.34).

274

275 For RT-qPCR analyses at specific developmental time points regardless of sex (Table 3), 276 Sdha and Pgk1 (GeoMean: 1.56 and 1.86) are best suited for analysis at E11.5. Following 277 determination of sex in the embryo at E12.5, RpL38 and Sdha with GeoMean values of 1.68 278 and 1.73 are recommended. Finally, RpL37 and Actb (GeoMean 1.86 and 1.86) are the best 279 suited for analysis of E15.5 brain tissue.

280

281 In comparison to a careful sex or stage approach above, we also pooled all the data 282 together (both sexes and all stages). Overall, this approached produced stability values 283 that were high (indicating a great variation between samples) for GeNorm and BestKeeper 284 packages (Supplementary Tables 5 and 6). The geometric mean of ranked references genes 285 for a pooled sample approached suggested that Sdha and RpL37 (Table 2 and 3), despite 286 287 $R p L 37$ often being ranked a poor choice if we were just consider the stabilities values for E11.5 and E12.5 individually (Supplementary Table 8 and Table 3). 
290 Previous micro-array data showed sex-specific expression of a number of genes within the

291 embryonic brain (forebrain) at E10.5 (Dewing et al., 2003). Wingless-type MMTV

292 integration site family, member $10 B($ Wnt10b) was shown to be up-regulated in male

293 embryos, with 1.7 fold increase in expression at E10.5 with respect to the female brain.

294 Cytochrome P450,7b1 (CYP7B1) was expressed 2.1 fold higher in the male brain, whereas

295 expression of X-inactive specific-transcript (Xist) was confirmed significantly higher in the

296 female embryo with a fold change of 18.5 compared to male samples. However, these

297 differences in gene expression were not studied over further time points, when

298 neurogenesis has commenced. To extend the findings from Dewing et al. (2003), regarding

299 sex-dimorphic expression of these genes, the top two ranked reference genes at each time

300 point from this study are used to compare against a selection of three genes in male and

301 female samples across embryonic brain development.

303 To normalize the expression of our three genes of interest, Sdha and Pgk1 oligonucleotide 304 primers were used at E11.5, RpL38 and Sdha were used for analysis for E12.5. For analysis 305 of E15.5, the reference genes Actb and RpL37 were used. Sex-dimorphic expression of Xist 306 was detected at all three time points with increased expression in female brain compared 307 to the males (Fig. 5A). CYP7B1 and Wnt10b also showed significant sex-dimorphic 308 expression at E11.5 $(P<0.05)$, with higher expression observed in the male brain at this 309 stage (Fig. 5C and 5E; 3.5-fold and 5-fold respectively). Additionally, following sex 310 determination, Wnt10b and CYP7B1 expression was not significantly different between the 311 sexes uses these stage-specific reference genes (Fig. 5C and 5E). In contrast, when pooling 312 all data from sex and age together the top two ranked genes were RpL37 and Sdha. When 
313 using these two genes to normalize expression data there is a large variation in the

314 expression of the three genes, especially at E12.5, between biological replicates (Fig. 5B, 5D

315 and 5F). No significant differences were observed in gene expression between male and

316 female samples, when using Pgk1 and Sdha as reference genes with the exception of Xist

317 expression at E12.5 (Fig. 5B, 5D and 4F).

318

319 When studying gene expression changes over time, two slightly different sets of reference

320 genes were ranked highest, depending upon the genotype of the sample (Table 2). We

321 normalized gene expression at each time point for female samples (with Sdha and Gapdh)

322 and for male samples (with $P g k 1$ and $S d h a$ ) to study how expression of these genes changes

323 with respect to developmental age (Fig. 6). In comparison, when $R p L 37$ (one of the poorer

324 stable genes for female and male time points) and Sdha where used as reference genes for

325 normalization of the all data points (top two genes when reference gene data was pooled

326 Table 2), this introduced so much variation between samples, any analysis would be

327 inconclusive (Supplementary Fig. 1).

328

329 CYP7B1 mRNA expression declines between E11.5 and E12.5 in both sexes but its 330 expression significantly increases again in the female only by E15.5 (Fig. 6A, P < 0.05). Xist

331 transcript expression in the female brain also increased between E11.5 and E15.5 332 (following sex-determination window (Fig. 1)) (Fig. 6B; $\mathrm{P}<0.001$ ). Gene expression of the

333 Wnt10b gene in the developing brain was low at all time-points and did not change 334 significantly overtime in the female staged embryo samples (Fig. 6C). However, the higher 
335 expression observed in the male embryonic samples decreased also between E11.5 and

336 E12.5, to similar expression levels found in XX samples (Fig. 6C).

338 Discussion

339 While studies using RTq-PCR have proven to be a powerful tool to study changes in gene 340 expression, there must be a careful consideration of the type of reference genes to be used.

341 As shown here, the reference genes used at particular developmental stages introduce 342 much more variation into the data analysis and is problematic for drawing conclusions 343 from RT-qPCR data. Therefore it is essential to determine use the best reference gene pair 344 for RT-qPCR analyses. For the comparison of sex-dimorphic gene expression at specific 345 time points, Sdha and Pgk1 are recommended at E11.5. At E12.5, Actb and Sdha are most 346 appropriate followed by Gapdh and Pgk1 are E15.5.

348 We determined the expression across time and between male and female sexes for three 349 genes, previously identified as being expressed sex-dimorphically prior to sex350 determination using the highest ranked references genes for each sex (Dewing et al., 2003).

351 Here we identified the ideal reference genes when studying male gene expression across 352 time, to be a combination of $A c t b$ and Sdha. In contrast, when studying gene expression 353 across developmental stage with female samples, Sdha and Gapdh would be most 354 appropriate. In comparison, if we used the Pgk1 gene (an X-linked gene) as the second 355 reference for female samples, this introduced a lot of variation between biological 356 replicates $(n=3$, pooled samples from different litters) at E11.5 and E12.5. This suggests 357 that expression of this commonly used reference gene was not stable in female embryonic 
358 brain tissues (Supp. Fig. 1). Pgk1 and Hprt are X-linked genes, subject to X inactivation

359 during cell differentiation (Chaumeil et al., 2006; Heard et al., 1997). This indicates that X-

360 linked genes should be avoided when analyzing gene expression in XX tissues samples at

361 early stages of brain development, particularly as our data for Xist (Fig. 6) indicates

362 changes to the expression of X-linked genes during brain development.

363

364 CYP7B1 converts the dihydrotesosterone (DHT) metabolite $5 \alpha$-androstane- $3 \beta, 17 \beta$-diol 365 (3ß-diol) to an inactive form and has been suggested to have a developmental role 366 maintaining normal levels of estrogens and androgens in the mammalian brain (Rose et al., 367 1997). CYP7B1 mRNA expression has been previously detected in the hippocampus, testis 368 and ovary (Wu et al., 1999). Knockout CYP7B1mice have an enlarged brain (late fetal stages 369 were examined) due to reduced apoptosis but overall brain weight normalizes after 370 puberty for both sexes (Sugiyama et al., 2009). In female mice CYP7B1 knockout results in 371 early onset puberty and ovarian failure (0moto et al., 2005). A recent study found that male 372 mutant have a reproductive behavioral defect, possibility due to altered olfactory cue 373 sensing (Oyola et al., 2015). We found that expression of CYP7B1 is sex-dimorphic at E11.5, 374 prior to any steroid hormone production by the developing gonads. By E15.5 the testis is 375 producing significant levels of testosterone and DHT, expression levels of CYP7B1 remained 376 low in the male but had increased in the female at E15.5 (Fig. 4C). Consistent with these 377 results, previously it was found that the human CYP7B1 promoter is suppressed by DHT in 378 human cell lines (Tang et al., 2006). In contrast, CYP7B1 expression increased with 379 overexpression of estrogen receptors. While in females there is no prenatal estradiol 380 production, 3ßAdiol, the target of CYP7B1 activity, is produced by the immature ovary 
381 (Sugiyama et al., 2010). 33Adiol binds to estrogen receptor $\beta$ (ER $\beta$ ), expression of this

382 receptor begins at E12.5, peaks at E18.5 and is active in the absence of estrogen, possibility

383 due to the presence of an alternative ligand such as 3ßAdiol (Sugiyama et al., 2010).

384 Together, this indicates that changes to the embryonic brain gene expression of CYP7B1

385 maybe regulated by androgen receptors and levels of steroid hormone metabolites during

386 mouse development. This may produce the changes we observed in CYP7B1 expression

387 across time in the female and males developing brain RNA samples (Fig. 4C and 5A).

388

389 The Wnt ligand, Wnt10b, was expressed at higher levels in male samples compared to 390 female E10.5 head samples in a previous microarray study (Dewing et al., 2003). While 391 Wnt10b knockout mice appear phenotypically normal, they exhibit a number of aging392 related phenotype such as bone loss (Stevens et al., 2010). Little has been studied 393 regarding the role of Wnt10b in early brain patterning, however research with the 394 zebrafish model indicated that Wnt10b functions redundantly with Wnt1 in specification of 395 the midbrain-hindbrain boundary (Lekven et al., 2003). Therefore it may play an early role 396 in patterning of the midbrain-hindbrain boundary in mice, but any functional consequence 397 for higher expression within the male developing brain at E10.5 (Dewing et al., 2003) and 398 E11.5 (Fig. 4E) requires further investigation.

400 Xist is an essential long non-coding RNA that has a role in gene dosage compensation in XX 401 embryos, by X chromosome in activation (XCI). During inactivation, the X chromosome that 402 will be inactivated up-regulates Xist expression, whereas Xist gene expression from the 403 active X chromosome is repressed (Galupa and Heard, 2015). We observed high levels of 
404 Xist expression in the female brain, compared to low or undetectable levels of Xist 405 expression in the males between E11.5-15.5 (Fig. 4A). Regulation of Xist gene expression is 406 complex, it is repressed by promoter bound CCCTC-binding factor (CTCF) and negatively 407 regulated by an anti-sense RNA, Tsix. A second ncRNA transcript, Jpx, expressed from both $408 \mathrm{X}$ chromosomes, binds to CTCF and removes it from the Xist promoter region promoting 409 expression of Xist (Sun et al., 2013). Xist gene expression (nor that of Jpx and Tsix) has not 410 been examined in any detail overtime nor in later developing tissues. The function of Xist 411 RNA in XCI is likely to be dosage sensitive (to Xist RNA levels) and tightly regulated. Mis412 regulation (up- or down) will allow either more genes to escape X-inactivation or result in a 413 reduction of expression of X-linked genes that are critical for brain development. 414 Overexpression of Xist has been linked to female psychiatric conditions in humans (Ji et al., 415 2015).

417 This study has shown that the optimal reference gene(s) varies with the sex and stage of 418 development. Therefore, when looking at gene expression either between sexes or across 419 time for a particular sex, careful consideration should be given to which reference gene(s) 420 are the most stable between the given samples and ideally use a combination of at least two 421 to provide the most robust results for data analysis.

422

\section{Acknowledgements}

424 We would like to thank Simon Blanchoud for the very helpful comments on the manuscript 425 drafts and Lisa Zondag for proofreading of the manuscript. 


\section{References}

427 Abel, K.M., Drake, R., and Goldstein, J.M. (2010). Sex differences in schizophrenia. Int Rev 428 Psychiatry 22, 417-428.

429 Andersen, C.L., Jensen, J.L., and Orntoft, T.F. (2004). Normalization of real-time quantitative 430 reverse transcription-PCR data: a model-based variance estimation approach to identify 431 genes suited for normalization, applied to bladder and colon cancer data sets. Cancer Res $43264,5245-5250$.

433 Arnold, A.P. (2004). Sex chromosomes and brain gender. Nat Rev Neurosci 5, 701-708.

434 Boda, E., Pini, A., Hoxha, E., Parolisi, R., and Tempia, F. (2009). Selection of reference genes 435 for quantitative real-time RT-PCR studies in mouse brain. J Mol Neurosci 37, 238-253.

436 Chaumeil, J., Le Baccon, P., Wutz, A., and Heard, E. (2006). A novel role for Xist RNA in the 437 formation of a repressive nuclear compartment into which genes are recruited when 438 silenced. Genes Dev 20, 2223-2237.

439 Chen, R.W., Saunders, P.A., Wei, H., Li, Z., Seth, P., and Chuang, D.M. (1999). Involvement of 440 glyceraldehyde-3-phosphate dehydrogenase (GAPDH) and p53 in neuronal apoptosis: 441 evidence that GAPDH is upregulated by p53. J Neurosci 19, 9654-9662.

442 Dewing, P., Shi, T., Horvath, S., and Vilain, E. (2003). Sexually dimorphic gene expression in 443 mouse brain precedes gonadal differentiation. Brain Res Mol Brain Res 118, 82-90.

444 Finlay, B.L., and Darlington, R.B. (1995). Linked regularities in the development and 445 evolution of mammalian brains. Science 268, 1578-1584.

446 Fombonne, E. (2009). Epidemiology of pervasive developmental disorders. Pediatr Res 65, 447 591-598.

448 Galupa, R., and Heard, E. (2015). X-chromosome inactivation: new insights into cis and 449 trans regulation. Curr Opin Genet Dev 31, 57-66.

450 Giedd, J.N., Castellanos, F.X., Rajapakse, J.C., Vaituzis, A.C., and Rapoport, J.L. (1997). Sexual 451 dimorphism of the developing human brain. Prog Neuropsychopharmacol Biol Psychiatry 452 21, 1185-1201.

453 Goldstein, J.M., Seidman, L.J., Horton, N.J., Makris, N., Kennedy, D.N., Caviness, V.S., Jr., 454 Faraone, S.V., and Tsuang, M.T. (2001). Normal sexual dimorphism of the adult human 455 brain assessed by in vivo magnetic resonance imaging. Cereb Cortex 11, 490-497. 
456 Gur, R.C., Turetsky, B.I., Matsui, M., Yan, M., Bilker, W., Hughett, P., and Gur, R.E. (1999). Sex 457 differences in brain gray and white matter in healthy young adults: correlations with 458 cognitive performance. J Neurosci 19, 4065-4072.

459 Heard, E., Clerc, P., and Avner, P. (1997). X-chromosome inactivation in mammals. Annu 460 Rev Genet 31, 571-610.

461 Hellemans, J., Mortier, G., De Paepe, A., Speleman, F., and Vandesompele, J. (2007). qBase 462 relative quantification framework and software for management and automated analysis of 463 real-time quantitative PCR data. Genome Biol 8, R19.

464 Hrdlickova, R., Toloue, M., and Tian, B. (2016). RNA-Seq methods for transcriptome 465 analysis. Wiley Interdiscip Rev RNA.

466 Ji, B., Higa, K.K., Kelsoe, J.R., and Zhou, X. (2015). Over-expression of XIST, the Master Gene 467 for X Chromosome Inactivation, in Females With Major Affective Disorders. EBioMedicine 468 2, 907-916.

469 Kouadjo, K.E., Nishida, Y., Cadrin-Girard, J.F., Yoshioka, M., and St-Amand, J. (2007). 470 Housekeeping and tissue-specific genes in mouse tissues. BMC Genomics 8, 127.

471 Lekven, A.C., Buckles, G.R., Kostakis, N., and Moon, R.T. (2003). Wnt1 and wnt10b function 472 redundantly at the zebrafish midbrain-hindbrain boundary. Dev Biol 254, 172-187.

473 Lenroot, R.K., Gogtay, N., Greenstein, D.K., Wells, E.M., Wallace, G.L., Clasen, L.S., Blumenthal, 474 J.D., Lerch, J., Zijdenbos, A.P., Evans, A.C., Thompson, P.M. and Giedd, J. N. (2007). Sexual 475 dimorphism of brain developmental trajectories during childhood and adolescence. 476 Neuroimage 36, 1065-1073.

477 Maekawa, F., Tsukahara, S., Kawashima, T., Nohara, K., and Ohki-Hamazaki, H. (2014). The 478 mechanisms underlying sexual differentiation of behavior and physiology in mammals and 479 birds: relative contributions of sex steroids and sex chromosomes. Front Neurosci 8, 242. 480 Mottron, L., Duret, P., Mueller, S., Moore, R.D., Forgeot d'Arc, B., Jacquemont, S., and Xiong, L. 481 (2015). Sex differences in brain plasticity: a new hypothesis for sex ratio bias in autism. Mol 482 Autism 6, 33.

483 Ngun, T.C., Ghahramani, N., Sanchez, F.J., Bocklandt, S., and Vilain, E. (2011). The genetics of 484 sex differences in brain and behavior. Front Neuroendocrinol 32, 227-246.

485 Omoto, Y., Lathe, R., Warner, M., and Gustafsson, J.A. (2005). Early onset of puberty and 486 early ovarian failure in CYP7B1 knockout mice. Proc Natl Acad Sci U S A 102, 2814-2819. 
487 Oyola, M.G., Zuloaga, D.G., Carbone, D., Malysz, A.M., Acevedo-Rodriguez, A., Handa, R.J., and 488 Mani, S.K. (2015). CYP7B1 Enzyme Deletion Impairs Reproductive Behaviors in Male Mice. 489 Endocrinology 156, 2150-2161.

490 Perrot-Sinal, T.S., Davis, A.M., and McCarthy, M.M. (2001). Developmental sex differences in 491 glutamic acid decarboxylase (GAD(65)) and the housekeeping gene, GAPDH. Brain Res 922, 492 201-208.

493 Pfaffl, M.W., Tichopad, A., Prgomet, C., and Neuvians, T.P. (2004). Determination of stable 494 housekeeping genes, differentially regulated target genes and sample integrity: 495 BestKeeper--Excel-based tool using pair-wise correlations. Biotechnol Lett 26, 509-515.

496 Rose, K.A., Stapleton, G., Dott, K., Kieny, M.P., Best, R., Schwarz, M., Russell, D.W., Bjorkhem, 497 I., Seckl, J., and Lathe, R. (1997). Cyp7b, a novel brain cytochrome P450, catalyzes the 498 synthesis of neurosteroids 7alpha-hydroxy dehydroepiandrosterone and 7alpha-hydroxy 499 pregnenolone. Proc Natl Acad Sci U S A 94, 4925-4930.

500 Saha, S., Chant, D., Welham, J., and McGrath, J. (2005). A systematic review of the prevalence 501 of schizophrenia. PLoS Med 2, e141.

502 Sawa, A., Khan, A.A., Hester, L.D., and Snyder, S.H. (1997). Glyceraldehyde-3-phosphate 503 dehydrogenase: nuclear translocation participates in neuronal and nonneuronal cell death. 504 Proc Natl Acad Sci U S A 94, 11669-11674.

505 Silver, N., Best, S., Jiang, J., and Thein, S.L. (2006). Selection of housekeeping genes for gene 506 expression studies in human reticulocytes using real-time PCR. BMC Mol Biol 7, 33.

507 Sotelo-Silveira, J., Crispino, M., Puppo, A., Sotelo, J.R., and Koenig, E. (2008). Myelinated 508 axons contain beta-actin mRNA and ZBP-1 in periaxoplasmic ribosomal plaques and 509 depend on cyclic AMP and F-actin integrity for in vitro translation. J Neurochem 104, 545510557.

511 Stevens, J.R., Miranda-Carboni, G.A., Singer, M.A., Brugger, S.M., Lyons, K.M., and Lane, T.F. 512 (2010). Wnt10b deficiency results in age-dependent loss of bone mass and progressive 513 reduction of mesenchymal progenitor cells. J Bone Miner Res 25, 2138-2147.

514 Stiles, J., and Jernigan, T.L. (2010). The basics of brain development. Neuropsychol Rev 20, $515 \quad 327-348$. 
516 Sugiyama, N., Andersson, S., Lathe, R., Fan, X., Alonso-Magdalena, P., Schwend, T., Nalvarte,

517 I., Warner, M., and Gustafsson, J.A. (2009). Spatiotemporal dynamics of the expression of

518 estrogen receptors in the postnatal mouse brain. Mol Psychiatry 14, 223-232, 117.

519 Sugiyama, N., Barros, R.P., Warner, M., and Gustafsson, J.A. (2010). ERbeta: recent

520 understanding of estrogen signaling. Trends Endocrinol Metab 21, 545-552.

521 Sun, S., Del Rosario, B.C., Szanto, A., Ogawa, Y., Jeon, Y., and Lee, J.T. (2013). Jpx RNA

522 activates Xist by evicting CTCF. Cell 153, 1537-1551.

523 Sur, M., and Rubenstein, J.L. (2005). Patterning and plasticity of the cerebral cortex. Science 524 310, 805-810.

525 Tang, W., Eggertsen, G., Chiang, J.Y., and Norlin, M. (2006). Estrogen-mediated regulation of 526 CYP7B1: a possible role for controlling DHEA levels in human tissues. J Steroid Biochem 527 Mol Biol 100, 42-51.

528 Veazey, K.J., and Golding, M.C. (2011). Selection of stable reference genes for quantitative 529 rt-PCR comparisons of mouse embryonic and extra-embryonic stem cells. PLoS One 6, 530 e27592.

531 Wu, Z., Martin, K.O., Javitt, N.B., and Chiang, J.Y. (1999). Structure and functions of human 532 oxysterol 7alpha-hydroxylase cDNAs and gene CYP7B1. J Lipid Res 40, 2195-2203.

533 Xie, F., Xiao, P., Chen, D., Xu, L., and Zhang, B. (2012). miRDeepFinder: a miRNA analysis tool 534 for deep sequencing of plant small RNAs. Plant Mol Biol. 80: 75.

535

536 


\section{Figure legends}

538 Figure 1: Brain developmental stages used in this study. In mice gonadal sex

539 determination occurs at $\sim$ E11.5. At E11.5 the neural tube has formed the primary brain

540 vesicles: Prosencephalon, Mesencephalon and Rhombencephalon. Between stages of E12.5

541 and E15.5, there is increased expansion of neuronal precursors and cell migration forming

542 cortical layers. Simultaneously, neurons differentiate to allow for axonal branching and

543 synapse formation.

544

545 Figure 2: Raw Ct values for selected reference genes from RT-qPCR using cDNA from

546 male and female brain tissue. (A) Raw Ct values for each reference gene are shown for

547 female and male samples by developmental stage. Data is plotted as floating bar plot, with 548 min and maximum values indicated and a line at the mean for each stage (E11.5, E12.5 and 549 E15.5).

550

551 Figure 3: Comparison of gene stability values for NormFinder and BestKeeper. (A) 552 NormFinder stability values for male and female reference genes across all developmental 553 stages. (B) The stability value of combined sexes calculated by NormFinder across all each 554 developmental time point of development. (C) The best suited reference genes for male and 555 female samples as calculated by BestKeeper shown as sdt error (+/- CP). (D) BestKeeper 556 values shown as std error (+/- CP) across time in samples of both sexes.

557 The lowest stability value for each sex is shown (Female, red; Male, blue; Both sexes, black). 558

559 Figure 4: Comparison of stability values for GeNorm and deltaCt methods. 
560 (A) Stability (M) value calculated by GeNorm across time in both male and female samples.

561 (B) GeNorm mean stability (M) values in male and female samples across all developmental

562 stages. (C) Average standard deviation using the deltaCt method for male, female and

563 combined samples. (D) Average standard deviation using the deltaCt method for each time

564 point tested.

565 The lowest stability value for each sex is shown (Female, red; Male, blue; Both sexes, black).

566

567 Figure 5: Sex specific gene expression using top ranked reference genes. (A) Xist

568 expression compared to the mean of the top two reference genes at each developmental

569 time point. (B) Xist expression compared to Pgk1 and Sdha reference genes at all stages of

570 development. (C) CYP7B1 expression compared to the mean of the top two reference genes

571 at each developmental time stage (D) CYP7B1 expression compared to Pgk1 and Sdha

572 reference genes at all stages of development. (E) Wnt10b expression compared to the mean

573 of top two reference genes at each developmental time point (F) Wnt10b expression

574 compared to Pgk1 and Sdha across all stages of development. Bar graphs are shown as the

575 mean of three replicates with error bars as the mean standard error of the mean. ${ }^{*}=\mathrm{P}<$ $5760.05^{* *}=\mathrm{P}<0.001^{* * *}=\mathrm{P}<0.0001$ (Student's unpaired T-test).

577

578 Figure 6. Expression of CYP7B1, Xist and Wnt10b across three stages of brain 579 development. (A). CYP7B1 mRNA expression in male (blue) and female (pink) brain tissue 580 across developmental stages E11.5, E12.5 and E15.5 normalised to the top ranked 581 reference genes for each sex over time. (B) Xist mRNA expression in male (blue) and 582 female (pink) brain tissue across developmental time stages normalized to the top ranked 
583 reference genes for each sex at E11.5, E12.5 and E15.5. (C) Wnt10b mRNA expression of 584 male (blue) and female (pink) brain tissue at developmental time stages E11.5, E12.5 and

585 E15.5. Data is show as mean and standard error of the mean. ${ }^{*}=\mathrm{P}<0.05^{* *}=\mathrm{P}<0.001$

586 Two-way ANOVA (Tukey's multiple comparison test). 
588 Table 1: Function, symbol and name of selected reference genes

\begin{tabular}{|l|l|l|}
\hline $\begin{array}{l}\text { Gene } \\
\text { symbol }\end{array}$ & Gene description & Function \\
\hline Gapdh & $\begin{array}{l}\text { Glyceraldehyde 3-phosphate } \\
\text { dehydrogenase }\end{array}$ & Catalyzes sixth step of glycolysis \\
\hline Actb & Beta-actin & Formation of microfilaments in eukaryotic cells \\
\hline Hprt1 & $\begin{array}{l}\text { Hypoxanthine guanine } \\
\text { phosphoribosyl transferase }\end{array}$ & $\begin{array}{l}\text { Transferase that plays a role in the generation of purine } \\
\text { nucleotide through the purine salvage pathway }\end{array}$ \\
\hline Pgk1 & Phosphoglycerate kinase 1 & $\begin{array}{l}\text { Part of the glycolysis pathway which catalyses the } \\
\text { conversion of 1,3-diphogycerate to 3-phosphoglycerate }\end{array}$ \\
\hline Sdha & $\begin{array}{l}\text { Succinate dehydrogenase complex, } \\
\text { subunit A, flavoprotein (Fp) }\end{array}$ & Citric acid cycle and the respiratory chain. \\
\hline Ppia & $\begin{array}{l}\text { Peptidylprolyl isomerase A } \\
\text { RpL38 }\end{array}$ & $\begin{array}{l}\text { Catalyzes cis-trans isomerization of proline imidic } \\
\text { peptide bonds. Role in protein folding. }\end{array}$ \\
\hline RpL37 & Ribosomal protein L38 & Protein synthesis \\
\hline Eif3f & $\begin{array}{l}\text { Eukaryotic translation initiation } \\
\text { factor 3 subunit F }\end{array}$ & Protein synthesis \\
\hline Eef2 & $\begin{array}{l}\text { Eukaryotic translation elongation } \\
\text { factor 2 }\end{array}$ & Translation elongation \\
\hline
\end{tabular}

Table 2. Geometric Mean of ranking values

591

\begin{tabular}{|l|l|l|l|l|l|l|}
\hline & Male & & Female & & \multicolumn{2}{|l|}{$\begin{array}{l}\text { All stages, male and } \\
\text { female }\end{array}$} \\
\hline 1 & Sdha & 1.96 & Sdha & 1.20 & Sdha & 1.18 \\
\hline 2 & Actb & 2.73 & Gapdh & 2.34 & RpL37 & 2.94 \\
\hline 3 & Pgk1 & 2.91 & Hprt1 & 2.45 & Actb & 3.03 \\
\hline 4 & Gapdh & 3.78 & RpL37 & 4.58 & Pgk1 & 3.10 \\
\hline 5 & RpL37 & 4.16 & Actb & 4.60 & Hprt1 & 3.83 \\
\hline 6 & Hprt1 & 4.64 & Pgk1 & 5.23 & RpL38 & 6 \\
\hline 7 & Actb & 4.82 & RpL38 & 5.63 & Gapdh & 6.34 \\
\hline 8 & Ppia & 7.95 & Ppia & 8.23 & Ppia & 7.23 \\
\hline 9 & Eef2 & 8.27 & Eef2 & 8.73 & Eef2 & 8.30 \\
\hline 10 & Eif3f & 10 & Eif3f & 10 & Eif3f & 10 \\
\hline
\end{tabular}

592 
594 Table 3. Geometric Mean of ranking values by developmental stage

\begin{tabular}{|l|l|l|l|l|l|l|}
\hline & E11.5 & & E12.5 & & E15.5 & \\
\hline 1 & Sdha & 1.56 & RpL38 & 1.68 & RpL37 & 1.86 \\
\hline 2 & Pgk1 & 1.86 & Sdha & 1.73 & Actb & 1.86 \\
\hline 3 & Actb & 2.91 & Pgk1 & 3.31 & Sdha & 3.31 \\
\hline 4 & Gapdh & 3.72 & Actb & 3.35 & Pgk1 & 3.72 \\
\hline 5 & RpL38 & 4.3 & RpL37 & 3.89 & Hprt1 & 3.86 \\
\hline 6 & RpL37 & 5.48 & Gapdh & 4.09 & Eef2 & 4.78 \\
\hline 7 & Hprt1 & 5.89 & Hprt1 & 5.95 & RpL38 & 6.70 \\
\hline 8 & Eef2 & 8.23 & Ppia & 7.44 & Gapdh & 7.02 \\
\hline 9 & Ppia & 9.14 & Eef2 & 7.77 & Ppia & 8.20 \\
\hline 10 & Eif3f & 9.24 & Eif3f & 10 & Eif3f & 10 \\
\hline
\end{tabular}

595

596

597 
598 Supplementary Table 1: Name, accession number and oligonucleotide primer sequences

599 used in this study.

\begin{tabular}{|l|l|l|l|}
\hline $\begin{array}{l}\text { Gene } \\
\text { symbol }\end{array}$ & $\begin{array}{l}\text { Accession } \\
\text { number }\end{array}$ & $\begin{array}{l}\text { Forward primer sequence (5'- } \\
\text { 3') }^{\prime}\end{array}$ & Reverse primer sequence (5'-3') \\
\hline Gapdh & NM_001289726 & TGTGTCCGTCGTGGATCTGA & CCTGCTTCACCACCTTCTTGA \\
\hline Actb & NM_007393.5 & GAGGTATCCTGACCCTGAAGTA & CACACGCAGCTCATTGTAGA \\
\hline Hprt1 & NM_013556.2 & CACAGGACTAGAACACCTGC & GCTGGTGAAAAGGACCTCT \\
\hline Pgk1 & NM_008828.3 & CTGACTTTGGACAAGCTGGACG & GCAGCCTTGATCCTTTGGTTG \\
\hline Sdha & NM_023281.1 & GCTCCTGCCTCTGTGGTTGA & AGCAACACCGATGAGCCTG \\
\hline Wnt10b & NM_011718.2 & TCCACTGGTGCTGTTATGTG & GCGAGGCTCACCTTCATTTA \\
\hline Xist & NR_001463.3 & GCCCAAAGGGACAAACAATC & GTAGCGAGGACTTGAAGAGAAG \\
\hline CYP7B1 & NP_004811.1 & CAGTGTCACAGGAAGTGAA & AGAGGAGAACACCCTCCTATT A \\
\hline Eef2 & NM_007907.2 & CTGGCAGAGGAC ATCGATAAG & GCAACGTCCCACTCATACTT \\
\hline RpL38 & NM_001048057. & $\begin{array}{l}\text { TTCGGTCTCATCGCTGTGAGTG } \\
\text { T }\end{array}$ & $\begin{array}{l}\text { TCTTGACAGACTTGGCATCCTTC } \\
\text { C }\end{array}$ \\
\hline Eif3f & NM_025344.2 & TTATTGTCGGCCAGGAGAAAG & GGGTGAGTCATGCCAACATA \\
\hline Ppia & NM_008907.1 & GTGTTCAGGGTGGTGACTTTA & AAGATGCCAGGACCTGTATG \\
\hline RpL37 & NM_026069.3 & CCAAGCGCAAGAGGAAGTATAA & CATGTCTGAATCTGCGGTAGAC \\
\hline Sry & NM_011564.1 & TCTTAAACTCTGAAGAAGAGAC & GTCTTGCCTGTATGTGATGG \\
\hline
\end{tabular}

600

601 Supplementary Table 2: Oligonucleotide primer efficiencies.

\begin{tabular}{|l|l|l|}
\hline Gene symbol & Efficiency & $\mathbf{R}^{\mathbf{2}}$ \\
\hline Gapdh & 100 & 0.997 \\
\hline Actb & 105 & 0.998 \\
\hline Hprt1 & 108 & 0.939 \\
\hline Pgk1 & 105 & 0.988 \\
\hline Sdha & 110 & 0.994 \\
\hline Wnt10b & 98 & 0.963 \\
\hline Xist & 91 & 0.993 \\
\hline CYP7B1 & 91 & 0.006 \\
\hline Eef2 & 108 & 0.993 \\
\hline Eif3f & 109 & 0.995 \\
\hline RpL38 & 92 & 0.963 \\
\hline RpL37 & 102 & 0.976 \\
\hline Ppia & 106 & 0.85 \\
\hline
\end{tabular}

602

603 
604 Supplementary Table 3. Mean Ct values with SD of each reference gene and

605 developmental stage

\begin{tabular}{|l|l|l|l|l|}
\hline & \multicolumn{1}{|l}{ E11.5 } & E12.5 & E15.5 & All stages \\
\hline Male & & & & \\
\hline Gapdh & $15.77(1.07)$ & $16.93(0.60)$ & $19.05(1.69)$ & $17.25(1.66)$ \\
\hline Actb & $15.92(1.38)$ & $15.46(0.42)$ & $17.69(1.13)$ & $16.36(1.17)$ \\
\hline Hprt1 & $22.68(1.51)$ & $22.81(1.18)$ & $25.57(1.25)$ & $23.69(1.63)$ \\
\hline Pgk1 & $20.30(1.37$ & $20.17(0.18)$ & $22.60(1.22)$ & $21.02(1.36)$ \\
\hline Sdha & $21.36(1.09)$ & $20.99(0.66)$ & $23.65(1.17)$ & $22.00(1.44)$ \\
\hline RpL37 & $16.63(1.18)$ & $19.31(2.30)$ & $20.32(1.50)$ & $18.75(2.24)$ \\
\hline RpL38 & $18.45(0.50)$ & $21.03(2.33)$ & $24.55(2.85)$ & $21.35(3.23)$ \\
\hline Eef2 & $19.27(1.97)$ & $23.84(5.70)$ & $27.83(1.26)$ & $23.65(4.82)$ \\
\hline Eif3f & $26.51(2.02)$ & $28.45(4.20)$ & $32.83(0.054)$ & $30.06(5.08)$ \\
\hline Ppia & $17.88(1.62)$ & $20.83(3.80)$ & $24.70(3.99)$ & $21.13(4.13)$ \\
\hline Female & \multicolumn{5}{|l|}{} & \\
\hline Gapdh & $17.00(4.35)$ & $17.00(2.25)$ & $22.08(5.46)$ & $18.69(2.93)$ \\
\hline Actb & $17.39(3.24)$ & $15.99(2.36)$ & $20.35(5.48)$ & $17.91(2.22)$ \\
\hline Hprt1 & $24.12(3.76)$ & $23.83(2.15)$ & $27.96(4.64)$ & $25.53(2.28)$ \\
\hline Pgk1 & $20.92(3.85)$ & $20.59(0.61)$ & $26.10(5.44)$ & $22.53(3.08)$ \\
\hline RpL37 & $15.8(0.49)$ & $18.78(2.05)$ & $19.39(3.12)$ & $17.99(2.51)$ \\
\hline RpL38 & $17.46(0.32)$ & $20.61(2.47)$ & $21.67(3.14)$ & $19.92(2.76)$ \\
\hline Sdha & $21.92(3.67)$ & $21.953(1.67)$ & $26.47(5.92)$ & $23.45(2.61)$ \\
\hline Eef2 & $17.67(1.32)$ & $24.46(4.85)$ & $24.03(3.79)$ & $22.05(4.54)$ \\
\hline Eif3f & $24.32(0.85)$ & $30.59(5.03)$ & $29.96(3.70)$ & $28.30(4.34)$ \\
\hline Ppia & $19.08(6.41)$ & $20.81(4.10)$ & $20.85(4.13)$ & $20.25(4.42)$ \\
\hline
\end{tabular}

606

607 
608 Supplementary Table 4: NormFinder stability values between male and females at each

609 stage. Shaded values are top genes with lowest stability value.

610

\begin{tabular}{|c|c|c|c|c|c|c|}
\hline & E11.5 & E12.5 & E15.5 & Male & Female & All stages \\
\hline ActB & 0.309 & 0.748 & 1.585 & 1.131 & 1.09 & 1.573 \\
\hline Hprt1 & 0.683 & 0.724 & 1.308 & 0.942 & 0.929 & 1.436 \\
\hline Sdha & 0.386 & 0.511 & 1.736 & 1.014 & 1.001 & 1.412 \\
\hline Gapdh & 0.511 & 0.806 & 1.809 & 0.932 & 1.099 & 1.446 \\
\hline Pgk1 & 0.461 & 0.691 & 1.847 & 1.049 & 1.079 & 1.548 \\
\hline Eef2 & 0.901 & 1.626 & 1.584 & 1.539 & 1.344 & 2.180 \\
\hline RpL38 & 0.830 & 0.253 & 1.835 & 0.862 & 1.0589 & 2.956 \\
\hline Eif3f & 1.031 & 1.968 & 2.924 & 1.671 & 2.162 & 3.781 \\
\hline Ppia & 1.156 & 1.011 & 2.121 & 1.289 & 1.525 & 1.856 \\
\hline RpL37 & 0.648 & 0.280 & 1.082 & 0.739 & 1.126 & 1.211 \\
\hline $\begin{array}{l}\text { Best two genes } \\
\text { (combined stability } \\
\text { value) }\end{array}$ & $\begin{array}{l}\text { Actb } \\
\text { and } \\
\text { Sdha } \\
(0.250)\end{array}$ & $\begin{array}{l}\text { Rp/38 } \\
\text { and } \\
\text { Rp/37 } \\
(0.189)\end{array}$ & $\begin{array}{l}\text { Pgk1 } \\
\text { and } \\
\text { Eef2 } \\
(0.430)\end{array}$ & $\begin{array}{l}\text { Hprt1 } \\
\text { and } \\
\text { Rp/38 } \\
\text { (0.559) }\end{array}$ & $\begin{array}{l}\text { Rpl38 } \\
\text { and } \\
\text { Sdha } \\
(0.640)\end{array}$ & $\begin{array}{l}\text { Rp/37 } \\
\text { and } \\
\text { Sdha } \\
(0.825)\end{array}$ \\
\hline
\end{tabular}

611

612 Supplementary Table 5: BestKeeper standard error (+/- CP) values between male and

613 females at each stage. Shaded values are top two genes with lowest std error values.

\begin{tabular}{|l|l|l|l|l|l|l|}
\hline & E11.5 & E12.5 & E15.5 & Male & Female & All stages \\
\hline ActB & 1.088 & 1.51 & 1.876 & 1.068 & 1.919 & 1.858 \\
\hline Hprt1 & 1.757 & 1.669 & 1.762 & 1.241 & 1.834 & 1.76 \\
\hline Sdha & 0.752 & 1.092 & 2.066 & 1.004 & 1.441 & 1.65 \\
\hline Gapdh & 0.934 & 1.529 & 2.295 & 1.226 & 1.639 & 1.783 \\
\hline Pgk1 & 0.71 & 0.522 & 2.379 & 1.064 & 1.827 & 1.846 \\
\hline Eef2 & 1.868 & 1.373 & 2.454 & 1.655 & 3.391 & 2.974 \\
\hline RpL38 & 0.686 & 0.901 & 2.699 & 1.62 & 1.706 & 2.559 \\
\hline Eif3f & 2.038 & 4.881 & 5.072 & 3.211 & 4.495 & 4.254 \\
\hline Ppia & 1.535 & 1.38 & 3.565 & 2.79 & 3.066 & 2.617 \\
\hline RpL37 & 1.032 & 1.248 & 1.52 & 1.237 & 1.702 & 1.514 \\
\hline
\end{tabular}

614

615 
617 Supplementary Table 6: GeNorm stability values between sexes at each stage. Mean M

618 value shaded top two ranked genes.

619

\begin{tabular}{|l|l|l|l|l|l|l|}
\hline & E11.5 & E12.5 & E15.5 & Male & Female & All stages \\
\hline ActB & 0.576 & 0.346 & 0.719 & 0.281 & 0.947 & 0.868 \\
\hline Hprt1 & 0.736 & 0.844 & 3.501 & 0.724 & 0.856 & 1.638 \\
\hline Sdha & 0.248 & 0.552 & 1.141 & 0.323 & 0.591 & 0.868 \\
\hline Gapdh & 0.448 & 0.346 & 4.592 & 0.559 & 0.591 & 3.546 \\
\hline Pgk1 & 0.248 & 1.045 & 0.719 & 0.281 & 1.062 & 1.067 \\
\hline Eef2 & 2.057 & 2.389 & 2.762 & 2.342 & 2.876 & 3.101 \\
\hline RpL38 & 1.807 & 1.47 & 2.935 & 1.617 & 1.85 & 2.571 \\
\hline Eif3f & 2.223 & 2.932 & 6.075 & 2.707 & 3.38 & 4.401 \\
\hline Ppia & 2.44 & 1.992 & 3.084 & 2.01 & 2.547 & 2.845 \\
\hline RpL37 & 1.46 & 1.659 & 2.239 & 1.195 & 2.212 & 2.29 \\
\hline
\end{tabular}

620

621 Supplementary Table 7: Average standard deviation (of delta Ct values) for reference 622 genes, sex and developmental stages.

623

\begin{tabular}{|l|l|l|l|l|l|l|}
\hline & E11.5 & E12.5 & E15.5 & Male & Female & $\begin{array}{l}\text { All } \\
\text { stages }\end{array}$ \\
\hline Gapdh & 2.09 & 2.5 & 8.16 & 2.29 & 2.82 & 5.13 \\
\hline Actb & 1.98 & 1.98 & 4.7 & 2.29 & 2.87 & 3.57 \\
\hline Hprt1 & 2.31 & 2.6 & 5.25 & 2.3 & 2.78 & 3.72 \\
\hline Pgk1 & 1.98 & 1.92 & 5.01 & 2.19 & 2.91 & 3.73 \\
\hline Sdha & 1.87 & 2.25 & 4.73 & 2.15 & 2.67 & 3.39 \\
\hline Eef2 & 2.76 & 4.02 & 5.17 & 3.51 & 4.14 & 4.71 \\
\hline RpL38 & 2.65 & 2.36 & 5.21 & 2.63 & 3.12 & 3.88 \\
\hline Eif3f & 3.01 & 5.11 & 12.01 & 4.17 & 5.44 & 7.82 \\
\hline Ppia & 3.31 & 3.08 & 5.54 & 3.14 & 3.92 & 4.3 \\
\hline RpL37 & 2.5 & 2.45 & 4.98 & 2.41 & 3.22 & 3.77 \\
\hline
\end{tabular}

624

625

626 
627 Supplementary Table 8: Summary of rankings across developmental stages by sex. 628

\begin{tabular}{lllllll}
\hline Ranking & Male & & \multicolumn{5}{c}{ Female } \\
\hline & BestKeeper & NormFinder & GeNorm & BestKeeper & NormFinder & GeNorm \\
$\mathbf{1}$ & Sdha & Rpl37 & Actb/Pgk1 & Sdha & Hprt1 & Sdha/Gapdh \\
$\mathbf{2}$ & Actb & Rpl38 & - & Gapdh & Sdha & - \\
$\mathbf{3}$ & Gapdh & Gapdh & Sdha & Rpl37 & Rpl38 & Hprt1 \\
$\mathbf{4}$ & Rpl37 & Hprt1 & Gapdh & Rpl38 & Actb & Actb \\
$\mathbf{5}$ & Hprt1 & Sdha & Hprt1 & Pgk1 & Gapdh & Pgk1 \\
$\mathbf{6}$ & Pgk1 & Pgk1 & Rpl37 & Hprt1 & Pgk1 & RpL38 \\
$\mathbf{7}$ & Eef2 & Actb & RpL38 & Actb & Rpl38 & RpL37 \\
$\mathbf{8}$ & Rpl38 & Ppia & Ppia & Ppia & Eef2 & Ppia \\
$\mathbf{9}$ & Ppia & Eef2 & Eef2 & Eef2 & Ppia & Eef2 \\
$\mathbf{1 0}$ & Eif3f & Eif3f & Eif3f & Eif3f & Eif3f & Eif3f \\
\hline
\end{tabular}

629

630 
631 Supplementary Table 8: Summary of rankings for comparing gene expression between

632 male and female samples at each embryonic stage.

\begin{tabular}{|c|c|c|c|}
\hline & BestKeeper & NormFinder & GeNorm \\
\hline \multicolumn{4}{|c|}{ E11.5 } \\
\hline 1 & Rpl38 & Actb & Sdha/Pgk1 \\
\hline 2 & Pgk1 & Sdha & - \\
\hline 3 & Sdha & Pgk1 & Gapdh \\
\hline 4 & Gapdh & Gapdh & Actb \\
\hline 5 & Rpl37 & Rpl37 & Hprt1 \\
\hline 6 & Actb & Hprt1 & RpL37 \\
\hline 7 & Ppia & Rpl38 & RpL38 \\
\hline 8 & Hprt & Eef2 & Eef2 \\
\hline 9 & Eef2 & Eif3f & Eif3f \\
\hline 10 & Eif3f & Ppia & Ppia \\
\hline \multicolumn{4}{|c|}{ E12.5 } \\
\hline 1 & Pgk1 & Rpl38 & Actb/Gapdh \\
\hline 2 & Rpl38 & Rpl37 & - \\
\hline 3 & Sdha & Sdha & Sdha \\
\hline 4 & Rpl37 & Pgk1 & Hprt1 \\
\hline 5 & Eef2 & Hprt1 & Pgk1 \\
\hline 6 & Ppia & $A c t b$ & RpL38 \\
\hline 7 & Actb & Gapdh & RpL37 \\
\hline 8 & Gapdh & Ppia & Ppia \\
\hline 9 & Hprt1 & Eef2 & Eef2 \\
\hline 10 & Eif3f & Eif3f & Eif3 \\
\hline \multicolumn{4}{|c|}{ E15.5 } \\
\hline 1 & Rpl37 & Rpl37 & Actb/Pgk1 \\
\hline 2 & Hprt1 & Hprt1 & - \\
\hline 3 & Actb & Eef2 & Sdha \\
\hline 4 & Sdha & $A c t b$ & RpL37 \\
\hline 5 & Gapdh & Sdha & Eef2 \\
\hline 6 & Pgk1 & Gapdh & RpL38 \\
\hline 7 & Eef2 & Rpl38 & Ppia \\
\hline 8 & Rpl38 & Pgk1 & Hprt1 \\
\hline 9 & Ppia & Ppia & Gapdh \\
\hline 10 & Eif3f & Eif3f & Eif3f \\
\hline
\end{tabular}


634 Supplementary Figure 1. Normalized gene expression for $C Y P 7 B 1$ (A), Wnt10b (B) and

635 Xist (C) using RpL37 and Sdha as combined reference genes. Normalized gene expression 636 for Wnt10b (D), Xist (E) and CYP7B1 using Pgk1 and Sdha as combined reference genes. 


\section{Figure 1}

Figure 1: Brain developmental stages used in this study.

In mice gonadal sex determination occurs at $\sim$ E11.5. At E11.5 the neural tube has formed the primary brain vesicles: Prosencephalon, Mesencephalon and Rhombencephalon. Between stages of E12.5 and E15.5, there is increased expansion of neuronal precursors and cell migration forming cortical layers. Simultaneously, neurons differentiate to allow for axonal branching and synapse formation.
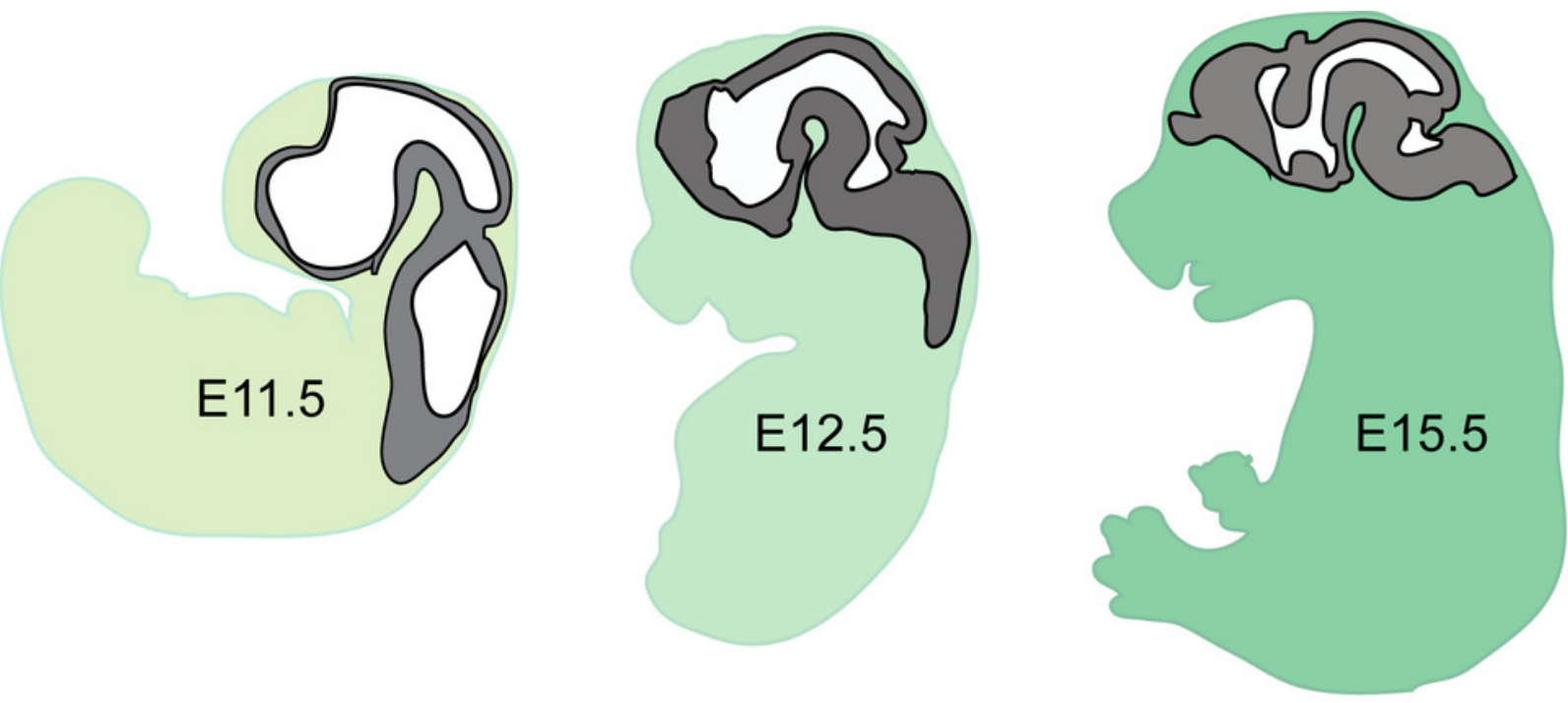

\begin{tabular}{|c|c|}
\hline $\begin{array}{c}\text { Patterning } \\
\text { of the neural tube }\end{array}$ & Neuronal development \\
\hline Sex Determination & nbryo - steroid hormone and AMH production \\
\hline
\end{tabular}


Figure 2

Figure 2: Raw Ct values for selected reference genes from RT-qPCR using cDNA from male and female brain tissue.
A) Raw Ct values for each reference gene are shown for female and male samples by developmental stage. Data is plotted as floating bar plot, with min and maximum values indicated and a line at the mean for each stage (E11.5, E12.5 and E15.5). 


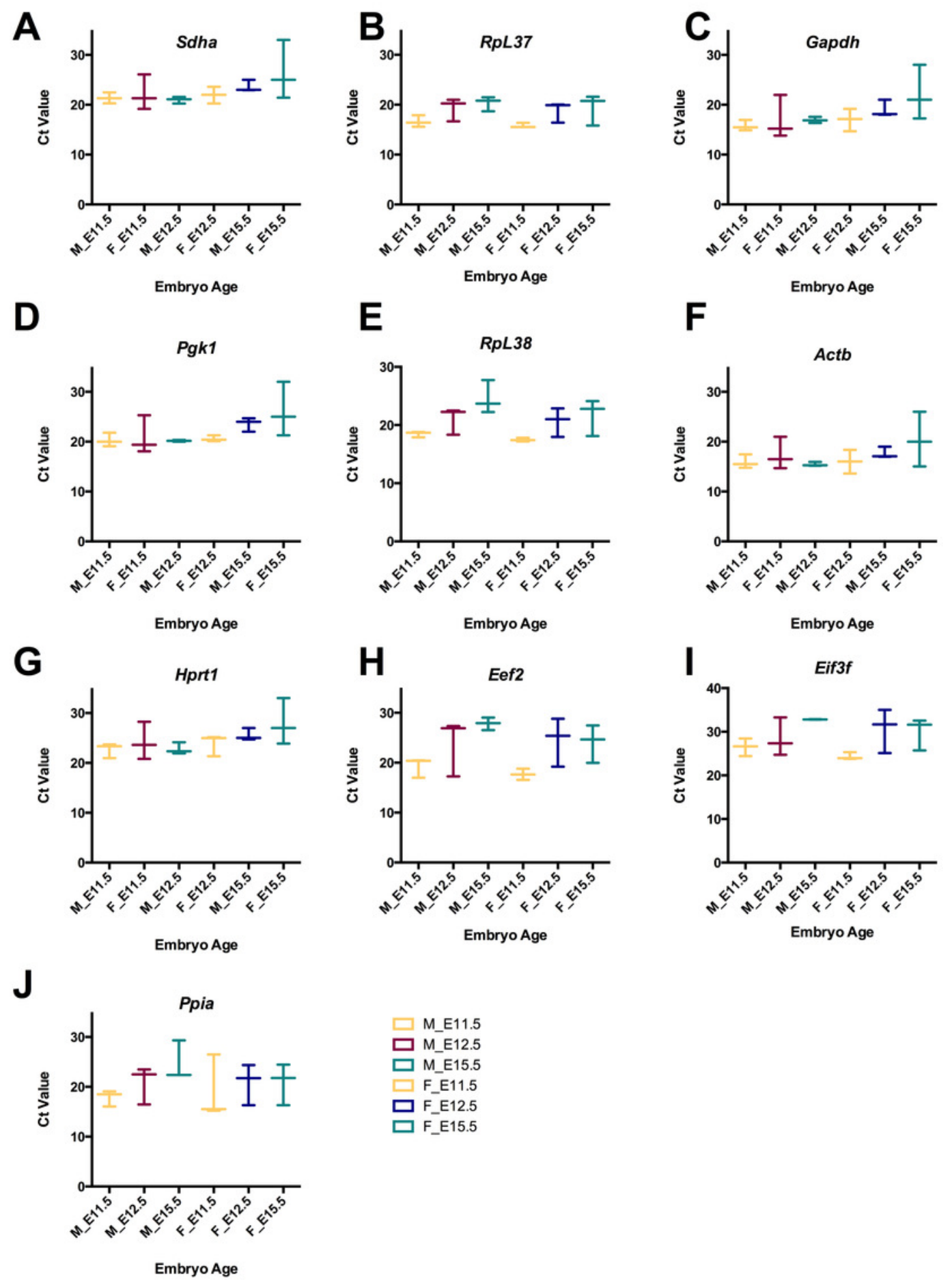




\section{Figure 3}

Figure 3: Comparison of gene stability values for NormFinder and BestKeeper.

(A) NormFinder stability values for male and female reference genes across all

developmental stages. (B) The stability value of combined sexes calculated by NormFinder across all each developmental time point of development. (C) The best suited reference genes for male and female samples as calculated by BestKeeper shown as sdt error (+/- CP). (D) BestKeeper values shown as std error (+/- CP) across time in samples of both sexes. The lowest stability value for each sex is shown (Female, red; Male, blue; Both sexes, black). 

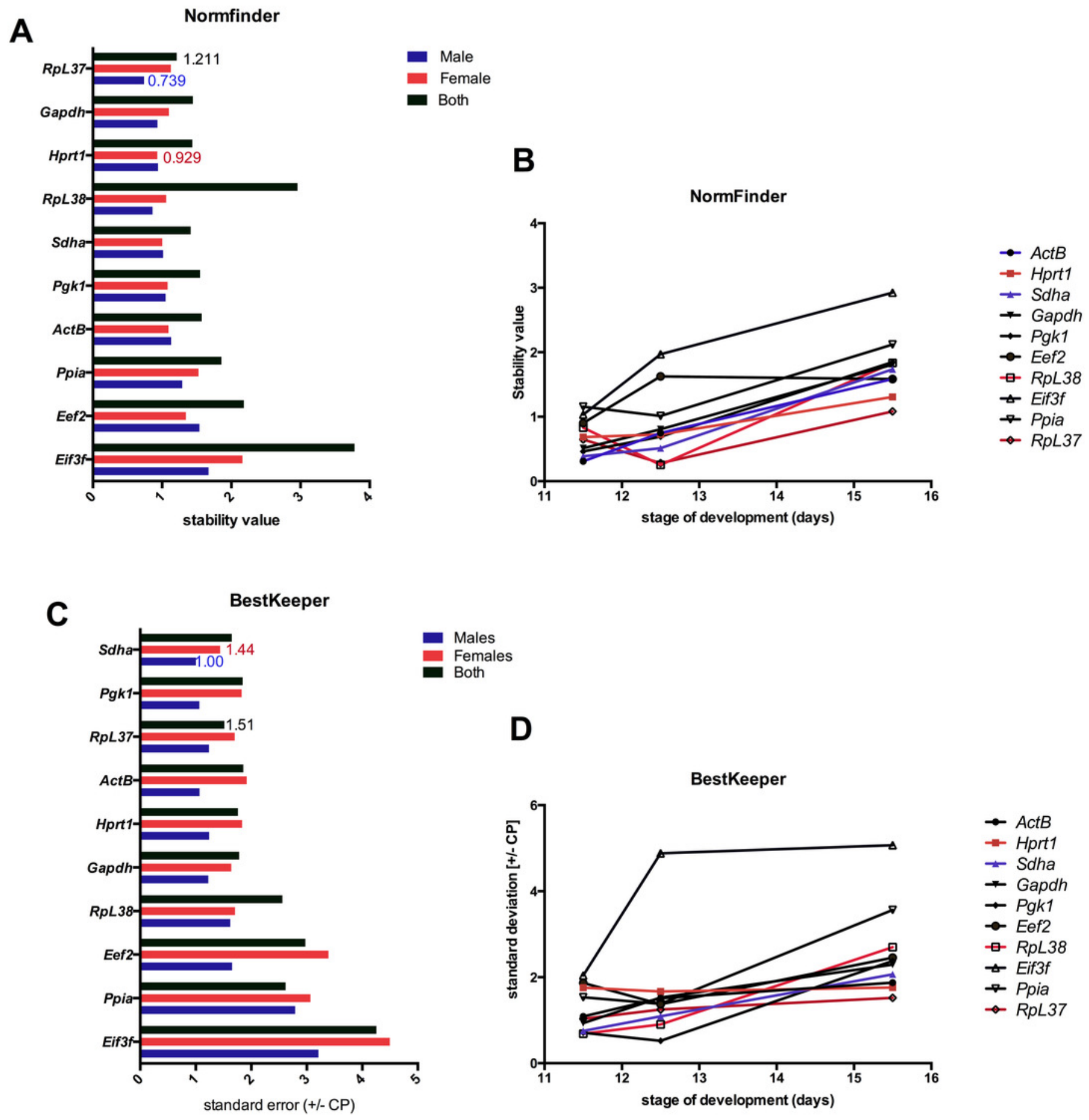


\section{Figure 4}

Figure 4: Comparison of stability values for GeNorm and deltaCt methods.

(A) Stability (M) value calculated by GeNorm across time in both male and female samples.

(B) GeNorm mean stability (M) values in male and female samples across all developmental stages. (C) Average standard deviation using the deltaCt method for male, female and combined samples. (D) Average standard deviation using the deltaCt method for each time point tested. The lowest stability value for each sex is shown (Female, red; Male, blue; Both sexes, black). 

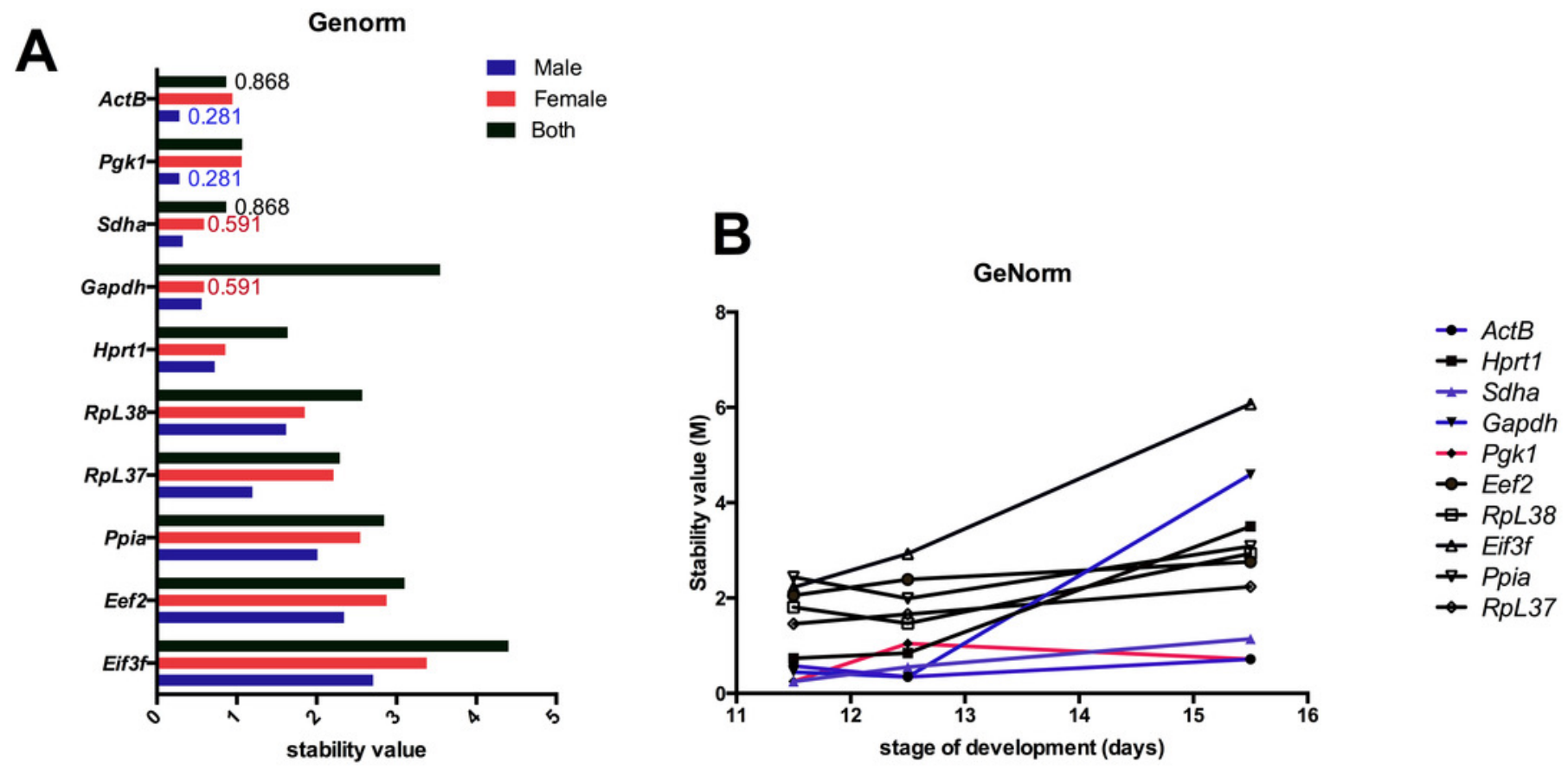

deltaCt
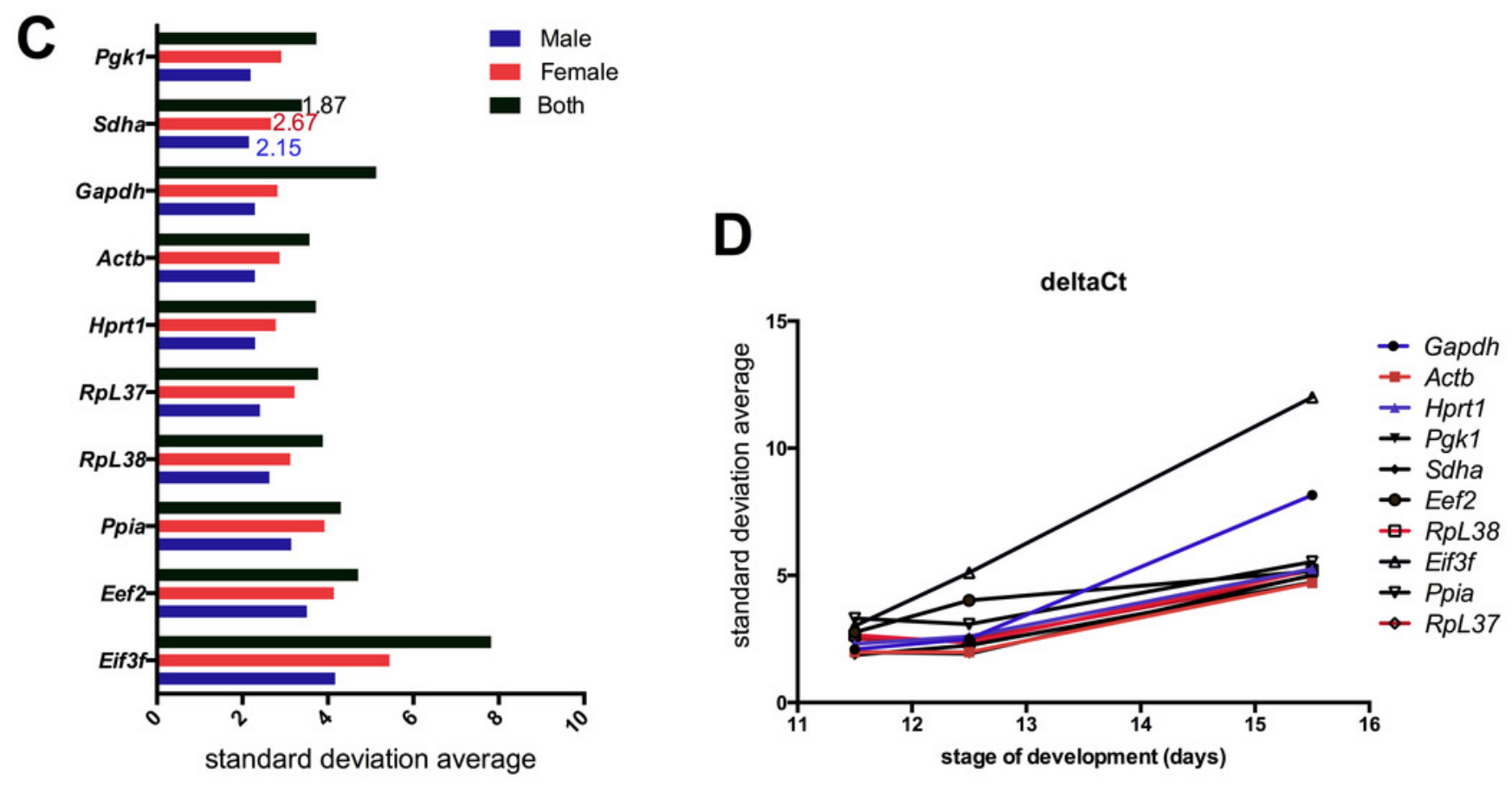


\section{Figure 5}

Figure 5: Sex specific gene expression using top ranked reference genes.

(A) Xist expression compared to the mean of the top two reference genes at each

developmental time point. (B) Xist expression compared to $P g k 1$ and Sdha reference genes at all stages of development. (C) CYP7B1 expression compared to the mean of the top two reference genes at each developmental time stage (D) CYP7B1 expression compared to Pgk1 and Sdha reference genes at all stages of development. (E) Wnt10b expression compared to the mean of top two reference genes at each developmental time point (F) Wnt10b expression compared to Pgk1 and Sdha across all stages of development. Bar graphs are shown as the mean of three replicates with error bars as the mean standard error of the mean. $*=P<0.05 * *=P<0.001 * * *=P<0.0001$ (Student's unpaired T-test). 
A

Xist

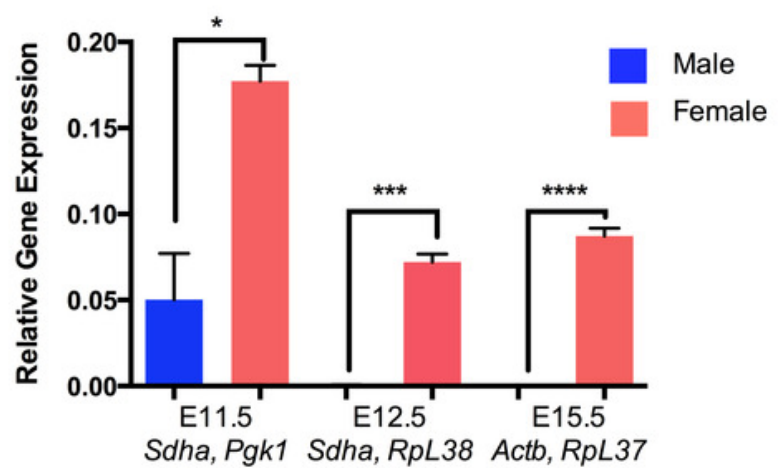

C

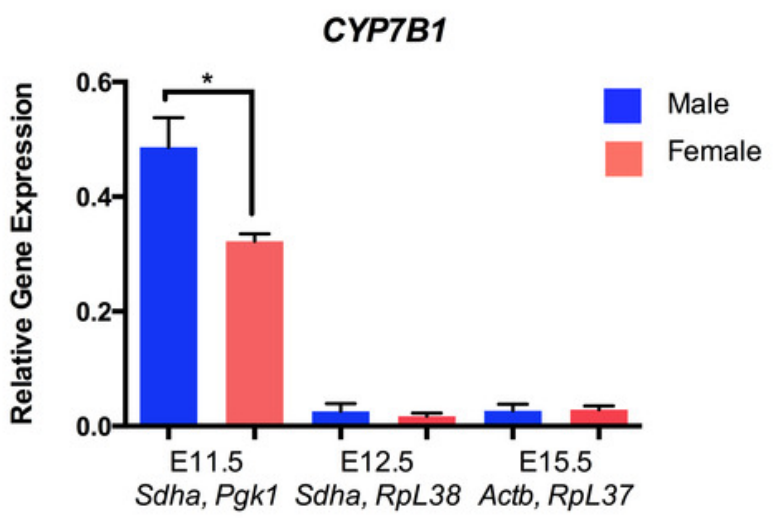

$\mathbf{E}$

Wnt10b

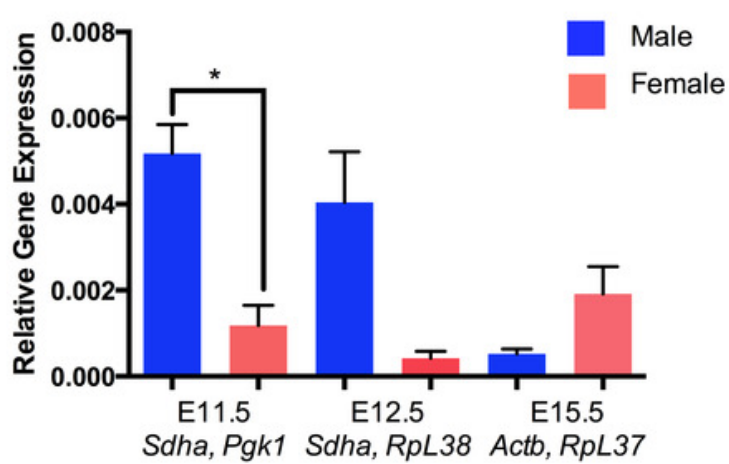

B

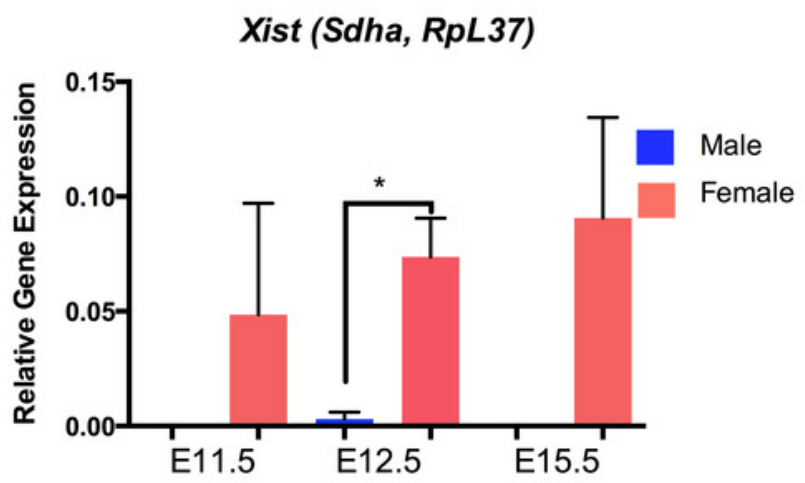

CYP7B1 (Sdha, RpL37)

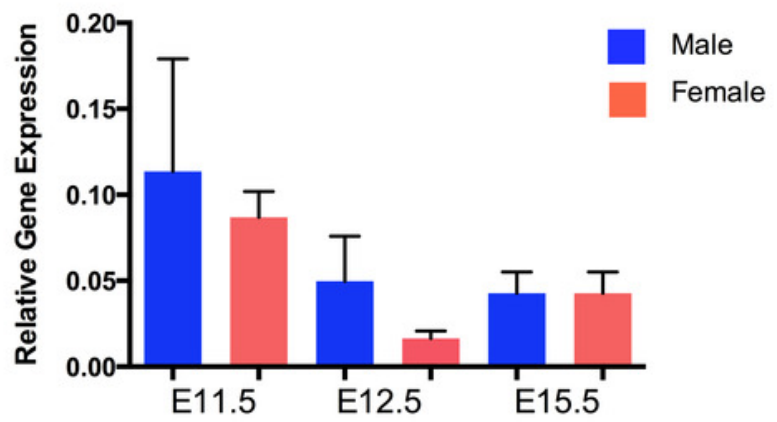

$\mathbf{F}$

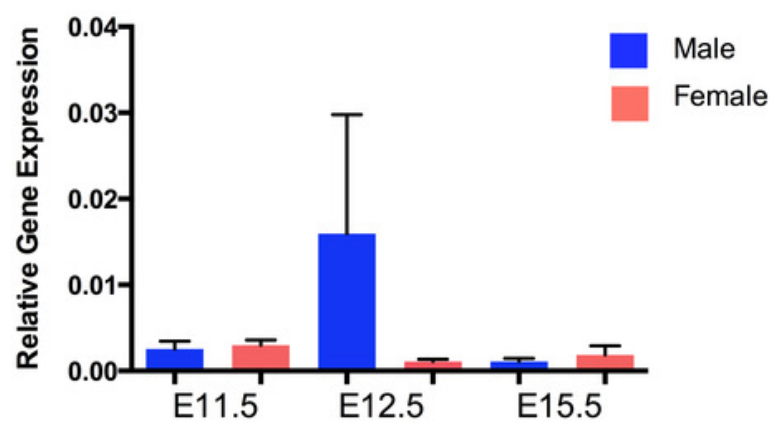




\section{Figure 6}

Figure 6. Expression of CYP7B1, Xist and Wnt10b across three stages of brain development.

(A). CYP7B1 mRNA expression in male (blue) and female (pink) brain tissue across developmental stages E11.5, E12.5 and E15.5 normalised to the top ranked reference genes for each sex over time. (B) Xist mRNA expression in male (blue) and female (pink) brain tissue across developmental time stages normalized to the top ranked reference genes for each sex at E11.5, E12.5 and E15.5. (C) Wnt10b mRNA expression of male (blue) and female (pink) brain tissue at developmental time stages E11.5, E12.5 and E15.5. Data is show as mean and standard error of the mean. ${ }^{*}=\mathrm{P}<0.05 * *=\mathrm{P}<0.001$ Two-way ANOVA (Tukey's multiple comparison test).

A

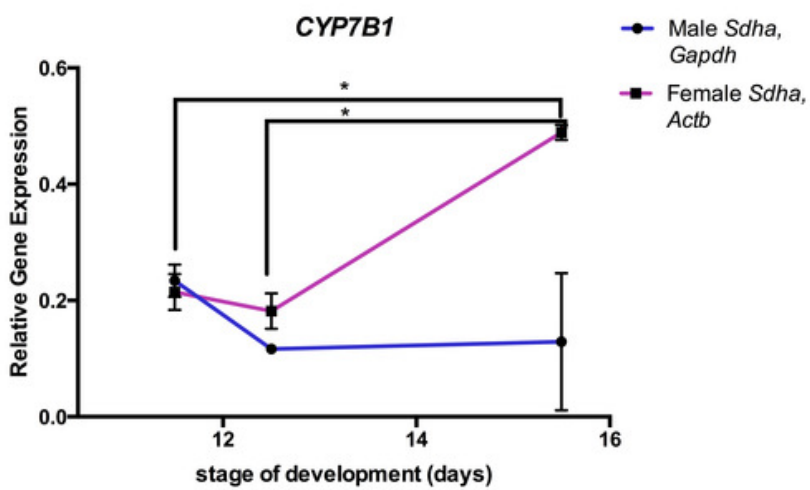

C

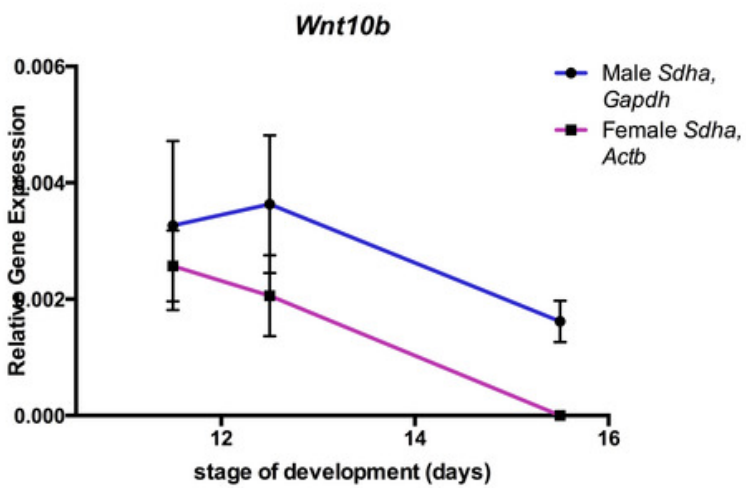

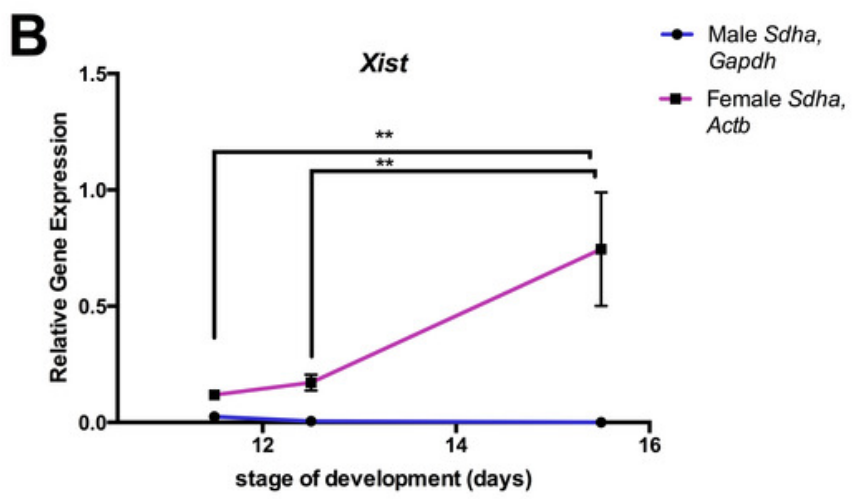




\section{Table 1 (on next page)}

Table 1: Function, symbol and name of selected reference genes 


\section{Table 1: Function, symbol and name of selected reference genes}

\begin{tabular}{|l|l|l|}
\hline $\begin{array}{l}\text { Gene } \\
\text { symbol }\end{array}$ & Gene description & Function \\
\hline Gapdh & $\begin{array}{l}\text { Glyceraldehyde 3-phosphate } \\
\text { dehydrogenase }\end{array}$ & Catalyzes sixth step of glycolysis \\
\hline Actb & Beta-actin & Formation of microfilaments in eukaryotic cells \\
\hline Hprt1 & $\begin{array}{l}\text { Hypoxanthine guanine } \\
\text { phosphoribosyl transferase }\end{array}$ & $\begin{array}{l}\text { Transferase that plays a role in the generation of purine } \\
\text { nucleotide through the purine salvage pathway }\end{array}$ \\
\hline Pgk1 & Phosphoglycerate kinase 1 & $\begin{array}{l}\text { Part of the glycolysis pathway which catalyses the } \\
\text { conversion of 1,3-diphogycerate to 3-phosphoglycerate }\end{array}$ \\
\hline Sdha & $\begin{array}{l}\text { Succinate dehydrogenase complex, } \\
\text { subunit A, flavoprotein (Fp) }\end{array}$ & Citric acid cycle and the respiratory chain. \\
\hline Ppia & Peptidylprolyl isomerase A & $\begin{array}{l}\text { Catalyzes cis-trans isomerization of proline imidic } \\
\text { peptide bonds. Role in protein folding. }\end{array}$ \\
\hline RpL38 & Ribosomal protein L38 & Protein synthesis \\
\hline RpL37 & Ribosomal protein L37 & Protein synthesis \\
\hline Eif3f & $\begin{array}{l}\text { Eukaryotic translation initiation } \\
\text { factor 3 subunit F }\end{array}$ & Translation elongation \\
\hline Eef2 & $\begin{array}{l}\text { Eukaryotic translation elongation } \\
\text { factor 2 }\end{array}$ & Translation elongation \\
\hline
\end{tabular}

2 
Table 2 (on next page)

Table 2. Geometric Mean of ranking values 
1 Table 2. Geometric Mean of ranking values

2

\begin{tabular}{|l|l|l|l|l|l|l|}
\hline & Male & & Female & \multicolumn{3}{|c|}{$\begin{array}{l}\text { All stages, male and } \\
\text { female }\end{array}$} \\
\hline 1 & Sdha & 1.96 & Sdha & 1.20 & Sdha & 1.18 \\
\hline 2 & Actb & 2.73 & Gapdh & 2.34 & RpL37 & 2.94 \\
\hline 3 & Pgk1 & 2.91 & Hprt1 & 2.45 & Actb & 3.03 \\
\hline 4 & Gapdh & 3.78 & RpL37 & 4.58 & Pgk1 & 3.10 \\
\hline 5 & RpL37 & 4.16 & Actb & 4.60 & Hprt1 & 3.83 \\
\hline 6 & Hprt1 & 4.64 & Pgk1 & 5.23 & RpL38 & 6 \\
\hline 7 & Actb & 4.82 & RpL38 & 5.63 & Gapdh & 6.34 \\
\hline 8 & Ppia & 7.95 & Ppia & 8.23 & Ppia & 7.23 \\
\hline 9 & Eef2 & 8.27 & Eef2 & 8.73 & Eef2 & 8.30 \\
\hline 10 & Eif3f & 10 & Eif3f & 10 & Eif3f & 10 \\
\hline
\end{tabular}

3

4 


\section{Table 3 (on next page)}

Table 3. Geometric Mean of ranking values by developmental stage 
1 Table 3. Geometric Mean of ranking values by developmental stage

\begin{tabular}{|l|l|l|l|l|l|l|}
\hline & E11.5 & & E12.5 & & E15.5 & \\
\hline 1 & Sdha & 1.56 & RpL38 & 1.68 & RpL37 & 1.86 \\
\hline 2 & Pgk1 & 1.86 & Sdha & 1.73 & Actb & 1.86 \\
\hline 3 & Actb & 2.91 & Pgk1 & 3.31 & Sdha & 3.31 \\
\hline 4 & Gapdh & 3.72 & Actb & 3.35 & Pgk1 & 3.72 \\
\hline 5 & RpL38 & 4.3 & RpL37 & 3.89 & Hprt1 & 3.86 \\
\hline 6 & RpL37 & 5.48 & Gapdh & 4.09 & Eef2 & 4.78 \\
\hline 7 & Hprt1 & 5.89 & Hprt1 & 5.95 & RpL38 & 6.70 \\
\hline 8 & Eef2 & 8.23 & Ppia & 7.44 & Gapdh & 7.02 \\
\hline 9 & Ppia & 9.14 & Eef2 & 7.77 & Ppia & 8.20 \\
\hline 10 & Eif3f & 9.24 & Eif3f & 10 & Eif3f & 10 \\
\hline
\end{tabular}

2 